\title{
Relation between parity-even and parity-odd CFT correlation functions in three dimensions
}

\author{
Sachin Jain and Renjan Rajan John \\ Indian Institute of Science Education and Research, \\ Homi Bhabha Road, Pashan, Pune 411 008, India \\ E-mail: sachin.jain@iiserpune.ac.in, \\ renjan.john@acads.iiserpune.ac.in
}

ABSTRACT: In this paper we relate the parity-odd part of two and three point correlation functions in theories with exactly conserved or weakly broken higher spin symmetries to the parity-even part which can be computed from free theories. We also comment on higher point functions.

The well known connection of CFT correlation functions with de-Sitter amplitudes in one higher dimension implies a relation between parity-even and parity-odd amplitudes calculated using non-minimal interactions such as $\mathcal{W}^{3}$ and $\mathcal{W}^{2} \widetilde{\mathcal{W}}$. In the flat-space limit this implies a relation between parity-even and parity-odd parts of flat-space scattering amplitudes.

KeYwords: Conformal Field Theory, Chern-Simons Theories

ARXiv EPRINT: 2107.00695 


\section{Contents}

1 Introduction 1

2 Relation between parity-even and parity-odd projection operators 3

3 Relation between parity-even and parity-odd two-point functions 5

3.1 Relation between parity-even and parity-odd in spinor-helicity variables 6

4 Generality: homogeneous (h) vs non-homogeneous (nh) parts of CFT $\begin{array}{ll}\text { correlators and amplitudes } & 6\end{array}$

$4.1 \mathrm{~h}$ vs nh in CFT correlation functions 6

$4.2 \mathrm{~h}$ vs nh in amplitudes 9

4.3 Summary of 3-point functions for exactly conserved currents 10

5 Relating parity-even and odd correlation function for exactly conserved currents 11

$\begin{array}{lll}5.1 & \text { Relation in spinor-helicity variables } & 12\end{array}$

$\begin{array}{ll}5.2 & \text { Relation in momentum space } \\ 5.3 & \text { Relation in }\end{array}$

5.3 Relation in position space 14

$\begin{array}{lll}5.4 & \text { Relation between parity-even and parity-odd three-point } d S_{4} \text { amplitude } & 15\end{array}$

$\begin{array}{lll}5.5 & \text { Four-point correlator } & 16\end{array}$

$\begin{array}{lll}5.5 .1 & \langle T O O O\rangle_{\text {even }} & 16\end{array}$

$\begin{array}{lll}5.5 .2\langle\text { TOOO }\rangle_{\text {odd }} & 17\end{array}$

6 A derivation using weakly broken higher spin symmetry 18

$\begin{array}{lll}6.1\left\langle T_{\mu \nu} J_{s_{1}} J_{s_{2}}\right\rangle & 19\end{array}$

$6.2\left\langle J_{s} T O\right\rangle \quad 21$

$\begin{array}{lll}6.3 & \langle\text { TOOO } & 21\end{array}$

$\begin{array}{lll}7 & \text { Summary and future directions } & 22\end{array}$

A Three-point function in momentum space 23

B Higher-spin correlators $\quad 28$

\section{Introduction}

Three dimensional conformal field theory finds diverse applications in topics from cosmology to condensed matter physics [1-7]. They also provide interesting examples of duality. For example, the ABJM super-conformal field theory is dual to $M$-theory on 
$A d S_{4} \times S^{7}[8,9]$. Another example is that of higher spin Vasiliev theories [10-13] which are dual to certain three-dimensional CFTs [14-16]. It also provides interesting examples of non-supersymmetric as well as supersymmetric dual pairs of field theory without gravity [17-20]. One interesting aspect of three dimensional CFT is that conformal invariance allows the correlation functions to have parity-odd contribution [21-23]. For example, the two-point functions of spinning operators have both parity-even and parity-odd contributions. The parity-even part can be reproduced from the free theory but the parity-odd contribution can only be obtained in interacting theories [17, 18, 24-26]. For three-point functions of exactly conserved currents, it was argued that in general there exist one parity-odd and two parity-even contributions [21]. The two parity-even structures are related to the free bosonic or free fermionic theory. However, the parity-odd contribution again requires us to consider interacting theories such as Chern-Simons matter theories [17, 18, 24-26]. ${ }^{1}$

The position space analysis of CFT correlation functions has attracted a great deal of attention [21-23, 27, 28]. However, much less attention has been devoted to understanding correlation function in momentum space or in spinor-helicity variables. See [29-41] and references therein for recent progress. For the spinor-helicity formalism in $A d S_{4}$, see [42]. Using results of [43] helicity structures of three-point correlators of higher-spin currents in momentum space and their relation with bulk AdS couplings were discussed in [44]. In momentum space using the light cone gauge, relations between the parity even and parity odd parts of a correlator can be observed from the results quoted in [44-46] which were given in light cone coordinates for specific kinematic regions. In [44], it was shown that the parity-breaking parameter $\theta$ results from certain EM duality in the bulk.

Recently it was realised that the parity-odd contribution to correlation functions of exactly conserved currents can be obtained from the parity-even contribution in spinorhelicity variables [40,41]. Using this result, it was shown in [47], that correlation functions in spinor-helicity variables in Chern-Simons matter theories can be written down using either free fermionic or free bosonic results. Naturally this poses the question, if this relation is special to spinor-helicity variables, or can one find out such relations in position space and momentum space as well? The aim of this note is to write down a precise relation between the parity-even and the parity-odd contributions to a correlator in position and momentum space. This relation in particular would imply that the parity-odd part of the correlation function of conserved currents can be obtained from free theory. In [25], the consistency of position space higher spin equations required a relation between the parity-odd and parityeven correlators for a few three-point correlators for specific components, but these were not explicitly checked. Recently in [40] a conformally invariant transformation that maps parity-even correlators to parity-odd correlators was constructed and this was explicitly checked at the level of two-point functions. ${ }^{2}$ In this paper we make use of the splitting of correlation functions into their homogeneous and non-homogeneous terms $[41,48]$ to relate

\footnotetext{
${ }^{1}$ The parity-odd part of $\left\langle J_{s} J_{s} O_{\Delta=2}\right\rangle$ can be obtained from the free fermionic theory in three dimensions. Similar conclusion holds for $\left\langle J_{s_{1}} J_{s_{2}} O_{\Delta=2}\right\rangle$. Interestingly, $\left\langle J_{s_{1}} J_{s_{2}} O_{\Delta=2}\right\rangle_{\text {even }}$ can only be obtained from interacting theories.

${ }^{2}$ We became aware of the results of [40] and the similarity with some of our results when we were done with the main results of our paper.
} 
the parity-even and the parity-odd correlators in position and momentum spaces as well as in spinor-helicity variables.

We will see that for correlation functions of exactly conserved currents with spins satisfying triangle inequality, the parity-odd contribution can be obtained from the homogeneous part of the parity-even contribution. One can think of this relation as a map from parity-even correlators to parity-odd correlators. For the non-homogeneous part, there is no analogous parity-odd contribution. When the triangle inequality is violated, there is no parity-odd contribution to the correlation function of exactly conserved currents. However, for weakly broken higher-spin theories, it turns out that such correlation functions can also have a parity-odd contribution. Interestingly, when the triangle inequality is violated the only contribution to the parity-odd and the parity-even parts is the non-homogeneous contribution. In this case as well, one can find out a relation between the parity-even and the parity-odd parts of the correlation function.

Rest of the paper is structured as follows. In section 2 we discuss the parity-even and the parity-odd projection operators that we use in this paper and the relation between them. In section 3 we discuss the relation between the parity-even and the parity-odd two-point functions of conserved currents of arbitrary spin $s$. In section 4 we describe the homogeneous and non-homogeneous contributions to 3-point functions comprising spinning operators in position space, momentum space and in spinor-helicity variables. We also describe these contributions to $d S_{4}$ amplitudes. In section 5 we describe the relation between the parity-even and the parity-odd homogeneous parts of three-point correlation functions of conserved currents, in spinor-helicity variables and in position and momentum spaces. To describe the relation in momentum space, we rewrite momentum space expressions using a few invariants such that the relation becomes manifest. We also extend our analysis to four-point functions. We then look at similar relations for three-point $d S_{4}$ amplitudes. In section 6 we use higher spin equations associated to weakly broken higher spin symmetry to derive the relation between parity-odd and parity-even correlators. We conclude with a summary and future directions of study in section 7. In appendix A we express three-point functions of conserved currents in terms of certain fundamental building blocks in momentum space. In appendix B we collect some details of higher spin equations that relate the parity-odd and the parity-even parts of correlators comprising higher spin currents.

\section{Relation between parity-even and parity-odd projection operators}

In this section we collect the orthogonal projectors that will be useful in the subsequent sections. We use the following parity-even projector to project spin-1 conserved currents

$$
\pi^{\mu \nu}(k)=\delta^{\mu \nu}-\frac{k^{\mu} k^{\nu}}{k^{2}}
$$


We can similarly define a parity-odd projector $\operatorname{as}^{3}$

$$
\chi^{\mu \nu}(k) \equiv \frac{1}{k} \epsilon^{\mu \nu k}
$$

where we have used the notation $\epsilon^{\mu \nu k} \equiv \epsilon^{\mu \nu \rho} k_{\rho}$. For spin-2 conserved currents, projectors are defined such that they are orthogonal and traceless. The parity-even and parity-odd projectors in this case are given by

$$
\begin{aligned}
& \Pi^{\mu \nu \rho \sigma}(k)=\frac{1}{2}\left(\pi^{\mu \rho}(k) \pi^{\nu \sigma}(k)+\pi^{\mu \sigma}(k) \pi^{\nu \rho}(k)-\pi^{\mu \nu}(k) \pi^{\rho \sigma}(k)\right) \\
& \Delta^{\mu \nu \rho \sigma}(k)=\frac{1}{4}\left[\epsilon^{\mu \rho k} \pi^{\nu \sigma}(k)+\epsilon^{\mu \sigma k} \pi^{\nu \rho}(k)+\epsilon^{\nu \sigma k} \pi^{\mu \rho}(k)+\epsilon^{\nu \rho k} \pi^{\mu \sigma}(k)\right]
\end{aligned}
$$

A relation between even and odd projectors. We observe the following relations between the parity-even and the parity-odd projectors defined above. For the spin-1 case, we have

$$
\frac{1}{k} \epsilon^{\mu \alpha k} \pi_{\alpha}^{\nu}(k)=\chi^{\mu \nu}(k), \quad \frac{1}{k} \epsilon^{\mu \alpha k} \chi_{\alpha}^{\nu}(k)=-\pi^{\mu \nu}(k)
$$

Similarly, for spin-2 projectors we have

$$
\epsilon^{\alpha k(\mu} \Pi_{\alpha}^{\nu) \rho \sigma}(k)=\Delta^{\mu \nu \rho \sigma}(k)
$$

where $(\mu, \nu)$ indicates symmetrisation with respect to $\mu$ and $\nu$ with a factor of $1 / 2$. It is also convenient to state these relations in terms of transverse, null polarization vectors which satisfy $k_{i} \cdot z_{i}=0$ and $z_{i}^{2}=0$. To do this, let us first write down the form of the projectors after contracting with these vectors

$$
\begin{array}{ll}
z_{1 \mu} z_{2 \nu} \pi^{\mu \nu}(k)=z_{1} \cdot z_{2} & z_{1 \mu} z_{1 \nu} z_{2 \rho} z_{2 \sigma} \Pi^{\mu \nu \rho \sigma}(k)=\left(z_{1} \cdot z_{2}\right)^{2} \\
z_{1 \mu} z_{2 \nu} \chi^{\mu \nu}(k)=\frac{1}{k} \epsilon^{z_{1} z_{2} k} & z_{1 \mu} z_{1 \nu} z_{2 \rho} z_{2 \sigma} \Delta^{\mu \nu \rho \sigma}(k)=\epsilon^{z_{1} z_{2} k}\left(z_{1} \cdot z_{2}\right)
\end{array}
$$

For the spin- $s$ case we have

$$
P_{s}^{\text {even }}=\left(z_{1} \cdot z_{2}\right)^{s}, \quad P_{s}^{\text {odd }}=\left(z_{1} \cdot z_{2}\right)^{s-1} \frac{1}{k} \epsilon^{z_{1} z_{2} k}
$$

We notice that the following substitution

$$
z_{1} \cdot z_{2} \rightarrow \frac{1}{k} \epsilon^{z_{1} z_{2} k}
$$

takes the parity-even projector to the parity-odd projector. More formally, the following operation

$$
\frac{1}{k} \epsilon^{z_{1} z_{2} k} \frac{1}{s} \frac{\partial}{\partial\left(z_{1} \cdot z_{2}\right)}: P_{s}^{\text {even }}(k) \rightarrow P_{s}^{\text {odd }}(k) .
$$

takes the parity-even projector to the parity-odd projector.

\footnotetext{
${ }^{3}$ While these are explicitly orthogonal to their momentum arguments they are different from usual projectors in the sense that they do not square to themselves. However, a contraction of three of these 'projectors' gives back the 'projector'.
} 


\section{Relation between parity-even and parity-odd two-point functions}

In this section we establish the relation between the parity-odd and the parity-even parts of two-point correlation functions in a generic 3d CFT. We will concern ourselves with only the momentum dependent structures and ignore constants. We start with the 2-point function of the spin-1 current.

$\langle\boldsymbol{J} \boldsymbol{J}\rangle$. The parity-even and parity-odd parts of the two-point function of the spin-1 current are given by

$$
\left\langle J^{\mu}(k) J^{\nu}(-k)\right\rangle_{\mathrm{even}}=\pi^{\mu \nu}(k) k, \quad\left\langle J^{\mu}(k) J^{\nu}(-k)\right\rangle_{\mathrm{odd}}=\epsilon^{\mu \nu k}
$$

One can easily check that the following relations hold

$$
\begin{aligned}
\frac{1}{k} \epsilon^{\mu \alpha k}\left\langle J_{\alpha}(k) J^{\nu}(-k)\right\rangle_{\text {even }} & =\left\langle J^{\mu}(k) J^{\nu}(-k)\right\rangle_{\text {odd }} \\
\frac{1}{k} \epsilon^{\mu \alpha k}\left\langle J_{\alpha}(k) J^{\nu}(-k)\right\rangle_{\text {odd }} & =-\left\langle J^{\mu}(k) J^{\nu}(-k)\right\rangle_{\text {even }}
\end{aligned}
$$

These relations can also be interpreted as the ones between parity-odd and parity-even projectors discussed in (2.5).

$\langle\boldsymbol{T} \boldsymbol{T}\rangle$. The above relation can be extended to the $\langle T T\rangle$ 2-point function. The parity-odd and the parity-even 2-point functions are given by

$$
\left\langle T^{\mu \nu}(k) T^{\rho \sigma}(-k)\right\rangle_{\mathrm{even}}=\Pi^{\mu \nu \rho \sigma}(k) k^{3} \quad\left\langle T^{\mu \nu}(k) T^{\rho \sigma}(-k)\right\rangle_{\mathrm{odd}}=\Delta^{\mu \nu \rho \sigma}(k) k^{2}
$$

In this case, we need to symmetrize as follows

$$
\frac{1}{2}\left[\frac{1}{k} \epsilon^{\mu \alpha k}\left\langle T^{\alpha \nu}(k) T^{\rho \sigma}(-k)\right\rangle_{\text {even }}+(\mu \leftrightarrow \nu)\right]=\left\langle T^{\mu \nu}(k) T^{\rho \sigma}(-k)\right\rangle_{\text {odd }}
$$

This is because the right hand side is symmetric under $(\mu \leftrightarrow \nu)$ by definition. Therefore, the left hand side must also be symmetric under this exchange.

To generalize this to higher spin currents, it is convenient to use polarization vectors. The parity-even and the parity-odd two-point functions of spin- 2 currents after contracting with polarization vectors are given by

$$
\begin{aligned}
z_{1 \mu} z_{1 \nu} z_{2 \rho} z_{2 \sigma}\left\langle T^{\mu \nu}(k) T^{\rho \sigma}(-k)\right\rangle_{\text {even }} & =\left(z_{1} \cdot z_{2}\right)^{2} k^{3} \\
z_{1 \mu} z_{1 \nu} z_{2 \rho} z_{2 \sigma}\left\langle T^{\mu \nu}(k) T^{\rho \sigma}(-k)\right\rangle_{\text {odd }} & =\epsilon^{z_{1} z_{2} k}\left(z_{1} \cdot z_{2}\right) k^{2}
\end{aligned}
$$

More generally

$$
\begin{aligned}
\left\langle z_{1}^{s} \cdot J_{s}(k) z_{2}^{s} \cdot J_{s}(-k)\right\rangle_{\text {even }} & =\left(z_{1} \cdot z_{2}\right)^{s} k^{2 s-1} \\
\left\langle z_{1}^{s} \cdot J_{s}(k) z_{2}^{s} \cdot J_{s}(-k)\right\rangle_{\text {odd }} & =\epsilon^{z_{1} z_{2} k}\left(z_{1} \cdot z_{2}\right)^{s-1} k^{2 s-2}
\end{aligned}
$$

where $z_{1}^{s}$ is shorthand notation for $z_{1}^{\mu_{1}} \cdots z^{\mu_{s}}$. We see that the map in (2.10) transforms the parity-even two-point function to the parity-odd two-point function. 


\subsection{Relation between parity-even and parity-odd in spinor-helicity variables}

All the statements made above can be seen manifestly in spinor-helicity variables in terms of which the 2-point function has a unique structure and the parity-even and parity-odd correlators differ only by a factor of $i{ }^{4}$ For the spin- $s$ current we have

$$
\begin{aligned}
& \left\langle J^{s-}\left(k_{1}\right) J^{s-}\left(k_{2}\right)\right\rangle=\left(c_{J_{s}}+i c_{J_{s}}^{\prime}\right) \frac{\langle 12\rangle^{2 s}}{2 s k_{2}}, \quad\left\langle J^{s+}\left(k_{1}\right) J^{s-}\left(k_{2}\right)\right\rangle=\left(c_{J_{s}}+i c_{J_{s}}^{\prime}\right) \frac{\langle\overline{1} 2\rangle^{2 s}}{2 s k_{2}} \\
& \left\langle J^{s+}\left(k_{1}\right) J^{s+}\left(k_{2}\right)\right\rangle=\left(c_{J_{s}}-i c_{J_{s}}^{\prime}\right) \frac{\langle\overline{1} \overline{2}\rangle^{2 s}}{2 s k_{2}}, \quad\left\langle J^{s-}\left(k_{1}\right) J^{s+}\left(k_{2}\right)\right\rangle=\left(c_{J_{s}}-i c_{J_{s}}^{\prime}\right) \frac{\langle 1 \overline{2}\rangle^{2 s}}{2 s k_{2}}
\end{aligned}
$$

We now turn our attention to three and higher point functions. To understand them, let us first explain a few important aspects of correlation functions.

We also discuss the relation between parity-even and the parity-odd two-point functions in position space in a later section.

\section{Generality: homogeneous (h) vs non-homogeneous (nh) parts of CFT correlators and amplitudes}

In this section we discuss the distinction between the homogeneous and the nonhomogeneous parts of correlation functions and amplitudes. This distinction will be useful in relating the parity-odd and parity-even parts of correlation functions.

\section{1 h vs nh in CFT correlation functions}

Any CFT correlation function can be separated into homogeneous and non-homogeneous parts, see [1] for a discussion in momentum space. In what follows we do this identification in momentum space, spinor-helicity variables and in position space.

Momentum space. The non-homogeneous part of the correlation function saturates the Ward-Takahashi (WT) identity, i.e.

$$
\begin{aligned}
k_{1 \mu_{1}}\left\langle J^{\mu_{1} \cdots \mu_{s_{1}}}\left(k_{1}\right) J_{s_{2}} J_{s_{3}} \cdots J_{s_{n}}\right\rangle_{\mathbf{h}} & =0 \\
k_{1 \mu_{1}}\left\langle J^{\mu_{1} \cdots \mu_{s_{1}}}\left(k_{1}\right) J_{s_{2}} J_{s_{3}} \cdots J_{s_{n}}\right\rangle_{\mathbf{n h}} & =\text { terms from the WT Identity }
\end{aligned}
$$

To be more concrete, let us consider the example of $\left\langle J_{\mu} J_{\nu} T_{\rho \sigma}\right\rangle$. The correlator can be written as

$$
\left\langle J_{\mu}\left(k_{1}\right) J_{\nu}\left(k_{2}\right) T_{\rho \sigma}\left(k_{3}\right)\right\rangle=\left\langle J_{\mu}\left(k_{1}\right) J_{\nu}\left(k_{2}\right) T_{\rho \sigma}\left(k_{3}\right)\right\rangle_{\text {Transverse }}+\left\langle J_{\mu}\left(k_{1}\right) J_{\nu}\left(k_{2}\right) T_{\rho \sigma}\left(k_{3}\right)\right\rangle_{\text {Local }} .
$$

When dotted with external momentum, the local piece reproduces the WT identity. The transverse piece can as well be split into homogeneous and non-homogeneous pieces

$$
\left\langle J_{\mu}\left(k_{1}\right) J_{\nu}\left(k_{2}\right) T_{\rho \sigma}\left(k_{3}\right)\right\rangle_{\text {Transverse }}=\left\langle J_{\mu}\left(k_{1}\right) J_{\nu}\left(k_{2}\right) T_{\rho \sigma}\left(k_{3}\right)\right\rangle_{\text {Trans,nh }}+\left\langle J_{\mu}\left(k_{1}\right) J_{\nu}\left(k_{2}\right) T_{\rho \sigma}\left(k_{3}\right)\right\rangle_{\text {Trans, h }}
$$

\footnotetext{
${ }^{4}$ For details of the spinor-helicity variables that we use in this paper we refer the reader to [1].
} 
Using the representation

$$
\left\langle J_{\mu}\left(k_{1}\right) J_{\nu}\left(k_{2}\right) T_{\rho \sigma}\left(k_{3}\right)\right\rangle=\left\langle J_{\mu}\left(k_{1}\right) J_{\nu}\left(k_{2}\right) T_{\rho \sigma}\left(k_{3}\right)\right\rangle_{\mathbf{n h}}+\left\langle J_{\mu}\left(k_{1}\right) J_{\nu}\left(k_{2}\right) T_{\rho \sigma}\left(k_{3}\right)\right\rangle_{\mathbf{h}}
$$

and comparing with (4.2) and (4.3) we obtain

$$
\begin{aligned}
\left\langle J_{\mu}\left(k_{1}\right) J_{\nu}\left(k_{2}\right) T_{\rho \sigma}\left(k_{3}\right)\right\rangle_{\mathbf{h}} & =\left\langle J_{\mu}\left(k_{1}\right) J_{\nu}\left(k_{2}\right) T_{\rho \sigma}\left(k_{3}\right)\right\rangle_{\text {Trans,h }} \\
\left\langle J_{\mu}\left(k_{1}\right) J_{\nu}\left(k_{2}\right) T_{\rho \sigma}\left(k_{3}\right)\right\rangle_{\mathbf{n h}} & =\left\langle J_{\mu}\left(k_{1}\right) J_{\nu}\left(k_{2}\right) T_{\rho \sigma}\left(k_{3}\right)\right\rangle_{\text {Trans,nh }}+\left\langle J_{\mu}\left(k_{1}\right) J_{\nu}\left(k_{2}\right) T_{\rho \sigma}\left(k_{3}\right)\right\rangle_{\text {Local }}
\end{aligned}
$$

It can also be shown easily by explicit computation that the homogeneous and nonhomogeneous pieces can be obtained from the free bosonic and the free fermionic theory as follows

$$
\begin{aligned}
\left\langle J_{\mu}\left(k_{1}\right) J_{\nu}\left(k_{2}\right) T_{\rho \sigma}\left(k_{3}\right)\right\rangle_{\mathbf{h}} & =\frac{1}{2}\left(\left\langle J_{\mu}\left(k_{1}\right) J_{\nu}\left(k_{2}\right) T_{\rho \sigma}\left(k_{3}\right)\right\rangle_{\mathrm{FB}}-\left\langle J_{\mu}\left(k_{1}\right) J_{\nu}\left(k_{2}\right) T_{\rho \sigma}\left(k_{3}\right)\right\rangle_{\mathrm{FF}}\right) \\
\left\langle J_{\mu}\left(k_{1}\right) J_{\nu}\left(k_{2}\right) T_{\rho \sigma}\left(k_{3}\right)\right\rangle_{\mathbf{n h}} & =\frac{1}{2}\left(\left\langle J_{\mu}\left(k_{1}\right) J_{\nu}\left(k_{2}\right) T_{\rho \sigma}\left(k_{3}\right)\right\rangle_{\mathrm{FB}}+\left\langle J_{\mu}\left(k_{1}\right) J_{\nu}\left(k_{2}\right) T_{\rho \sigma}\left(k_{3}\right)\right\rangle_{\mathrm{FF}}\right)
\end{aligned}
$$

Spinor-helicity variables. It is easiest to distinguish between homogeneous and nonhomogeneous contributions in spinor-helicity variables. In spinor-helicity variables, the action of the special conformal generator on the homogeneous piece gives zero whereas on the non-homogeneous piece it gives the terms that appear in the WT identity. It is also the case that at the level of three-point functions, homogeneous and non-homogeneous contributions have different pole structures in $E=k_{1}+k_{2}+k_{3}$. Generically the homogeneous contribution is always more singular. As an example, let us consider the parity-even part of the stress-tensor three-point function $[30,49]$ in spinor-helicity variables

$$
\begin{aligned}
\left\langle T^{-} T^{-} T^{-}\right\rangle_{\text {even }} & =\left(c_{1} \frac{c_{123}}{E^{6}}+c_{T} \frac{E^{3}-E b_{123}-c_{123}}{c_{123}^{2}}\right)\langle 12\rangle^{2}\langle 23\rangle^{2}\langle 31\rangle^{2} \\
\left\langle T^{-} T^{-} T^{+}\right\rangle_{\text {even }} & =c_{T} \frac{\left(E-2 k_{3}\right)^{2}\left(E^{3}-E b_{123}-c_{123}\right)}{E^{2} c_{123}^{2}}\langle 12\rangle^{2}\langle 2 \overline{3}\rangle^{2}\langle\overline{3} 1\rangle^{2}
\end{aligned}
$$

where $c_{123}=k_{1} k_{2} k_{3}$ and $b_{123}=\left(k_{1} k_{2}+k_{2} k_{3}+k_{3} k_{1}\right)$. Note that $c_{T}$ comes from the parityeven two-point function of the stress tensor (3.8). The term proportional to $c_{1}$ is the homogeneous contribution whereas the term proportional to $c_{T}$ is the non-homogeneous contribution. It is clear that the pole structure in $E$ of the homogeneous and non-homogeneous pieces are different. Let us also emphasize that the homogeneous piece contributes only to the --- and +++ helicity components whereas the non-homogeneous piece contributes to all helicity components.

Position space. Let us now distinguish between the homogeneous and non-homogeneous contributions in position space. We consider two examples to illustrate the distinction.

$\left\langle\boldsymbol{J}\left(\boldsymbol{x}_{1}\right) \boldsymbol{J}\left(\boldsymbol{x}_{2}\right) \boldsymbol{O}_{\Delta}\left(x_{3}\right)\right\rangle$. The WT identity for correlation functions of the form $\left\langle J\left(x_{1}\right) J\left(x_{2}\right) O_{\Delta}\left(x_{3}\right)\right\rangle$ is given by

$$
\partial_{1 \mu}\left\langle J^{\mu}\left(x_{1}\right) J^{\nu}\left(x_{2}\right) O_{\Delta}\left(x_{3}\right)\right\rangle=0
$$


which implies that the correlator has only a homogeneous part. In terms of certain conformal structures the parity-even and the parity-odd parts of the correlator can be expressed as follows [21]

$$
\begin{aligned}
\left\langle J_{\mu}\left(x_{1}\right) J_{\nu}\left(x_{2}\right) O_{\Delta}\left(x_{3}\right)\right\rangle_{\text {even, } \mathbf{h}} & =\frac{1}{\left|x_{12}\right|^{2-\Delta}\left|x_{23}\right|^{\Delta}\left|x_{31}\right|^{\Delta}}\left(\Delta Q_{1} Q_{2}+(4-2 \Delta) P_{3}^{2}\right) \\
\left\langle J_{\mu}\left(y_{1}\right) J_{\nu}\left(y_{2}\right) O_{\Delta}\left(y_{3}\right)\right\rangle_{\text {odd, } \mathbf{h}} & =\frac{1}{\left|x_{12}\right|^{2-\Delta}\left|x_{23}\right|^{\Delta}\left|x_{31}\right|^{\Delta}} S_{3} .
\end{aligned}
$$

For details of the notation see [21].

Let us consider another example $\left\langle T\left(x_{1}\right) J\left(x_{2}\right) J\left(x_{3}\right)\right\rangle$. The correlator is given by [21]

$$
\begin{gathered}
\left\langle T\left(x_{1}\right) J\left(x_{2}\right) J\left(x_{3}\right)\right\rangle_{\text {even }}=\frac{1}{\left|x_{12}\right|\left|x_{23}\right|\left|x_{31}\right|} \\
{\left[c_{1}\left(P_{1}^{2} Q_{1}^{2}-4 P_{2}^{2} P_{3}^{2}-\frac{5}{2} Q_{1}^{2} Q_{2} Q_{3}-2 P_{1} P_{2} P_{3} Q_{1}\right)\right.} \\
\left.+c_{j} P_{1} P_{2} P_{3} Q_{1}\right]
\end{gathered}
$$

where $c_{j}$ is the coefficient of the two-point function of $J_{\mu}$ fixed by the WT identity. The term proportional to $c_{1}$ is homogeneous and the term proportional to $c_{j}$ is the non-homogeneous contribution. One can always add a homogeneous piece to the non-homogeneous piece of the correlator. Doing this, one obtains

$$
\begin{aligned}
\left\langle T\left(x_{1}\right) J\left(x_{2}\right) J\left(x_{3}\right)\right\rangle_{\text {even }}=\frac{1}{\left|x_{12}\right|\left|x_{23}\right|\left|x_{31}\right|} & {\left[c_{1}\left(P_{1}^{2} Q_{1}^{2}-4 P_{2}^{2} P_{3}^{2}-\frac{5}{2} Q_{1}^{2} Q_{2} Q_{3}-2 P_{1} P_{2} P_{3} Q_{1}\right)\right.} \\
+ & \left.c_{j}\left(P_{1}^{2} Q_{1}^{2}-4 P_{2}^{2} P_{3}^{2}-\frac{5}{2} Q_{1}^{2} Q_{2} Q_{3}+2 P_{1} P_{2} P_{3} Q_{1}\right)\right]
\end{aligned}
$$

We observe that in the representation (4.11), the homogeneous and the non-homogeneous pieces can be obtained from each other by taking $P_{3} \rightarrow-P_{3}$.

One can write down a general polynomial for the parity-even homogeneous and non-homogeneous contributions to correlation functions involving general conserved currents as follows

$$
\begin{gathered}
\mathcal{F}_{\mathbf{h}}=e^{\frac{Q_{1}+Q_{2}+Q_{3}}{2} e^{P_{1}+P_{2}-P_{3}}} \\
\mathcal{F}_{\mathbf{n h}}=e^{\frac{Q_{1}+Q_{2}+Q_{3}}{2}} e^{P_{1}+P_{2}+P_{3}} .
\end{gathered}
$$

As is clear we have

$$
P_{3} \rightarrow-P_{3}: \mathcal{F}_{\mathbf{h}} \rightarrow \mathcal{F}_{\mathbf{n h}}
$$

It would be interesting to find out if there exists a similar relation between homogeneous and non-homogeneous contributions in momentum space. It is useful to note the following representation of three-point correlation functions in the free bosonic and free fermionic theories

$$
\begin{aligned}
& \mathcal{F}_{\mathrm{FB}}=\mathcal{F}_{\mathbf{n h}}+\mathcal{F}_{\mathbf{h}}=e^{\frac{Q_{1}+Q_{2}+Q_{3}}{2}} e^{P_{1}+P_{2}}\left(e^{P_{3}}+e^{-P_{3}}\right) \\
& \mathcal{F}_{\mathrm{FF}}=\mathcal{F}_{\mathbf{n h}}-\mathcal{F}_{\mathbf{h}}=e^{\frac{Q_{1}+Q_{2}+Q_{3}}{2}} e^{P_{1}+P_{2}}\left(e^{P_{3}}-e^{-P_{3}}\right) .
\end{aligned}
$$


which precisely matches the representation given in equations 15 and 16 of [50]. Let us note that (4.14) implies

$$
\begin{aligned}
& P_{3} \rightarrow-P_{3}: \mathcal{F}_{\mathrm{FB}} \rightarrow \mathcal{F}_{\mathrm{FB}} \\
& P_{3} \rightarrow-P_{3}: \mathcal{F}_{\mathrm{FF}} \rightarrow-\mathcal{F}_{\mathrm{FF}} .
\end{aligned}
$$

We can express the homogeneous and the non-homogeneous pieces in terms of the free boson and free fermion answers as follows

$$
\begin{aligned}
\mathcal{F}_{\mathbf{h}} & =\frac{1}{2}\left(\mathcal{F}_{\mathrm{FB}}-\mathcal{F}_{\mathrm{FF}}\right) \\
\mathcal{F}_{\mathbf{n h}} & =\frac{1}{2}\left(\mathcal{F}_{\mathrm{FB}}+\mathcal{F}_{\mathrm{FF}}\right) .
\end{aligned}
$$

A simple way to understand (4.16) is the following

$$
\begin{aligned}
\partial_{1 \mu_{1}}\left\langle J^{\mu_{1} \cdots \mu_{s_{1}}}\left(x_{1}\right) J_{s_{2}} J_{s_{3}}\right\rangle_{\mathbf{h}} & =\partial_{1 \mu_{1}}\left(\left\langle J^{\mu_{1} \cdots \mu_{s_{1}}}\left(x_{1}\right) J_{s_{2}} J_{s_{3}}\right\rangle_{\mathbf{F B}}-\left\langle J^{\mu_{1} \cdots \mu_{s_{1}}}\left(x_{1}\right) J_{s_{2}} J_{s_{3}}\right\rangle_{\mathbf{F F}}\right) \\
& =\text { FB WT Identity }- \text { FF WT Identity } \\
& =0
\end{aligned}
$$

where in the last step we have identified the WT identity for the bosonic and fermionic theories. This identification requires us to identify the two-point function of boson and fermion. This also fixes the relative normalization. We emphasise that in this section we have considered correlators that satisfy triangle inequality (4.25). Outside the triangle inequality all the contributions are non-homogeneous and we discuss them in the later sections.

\section{2 h vs nh in amplitudes}

We will now distinguish between the homogeneous and non-homogeneous contributions to amplitudes. As we shall see, the gravity amplitude automatically comes in a way which separates out the homogeneous and non-homogeneous contributions. For this purpose, let us consider the $n$-point amplitude $\mathcal{M}^{\mu_{1}, \mu_{2} \cdots \mu_{s_{1}+s_{2} \cdots s_{n}}}\left(k_{1}, k_{2} \cdots, k_{n}\right)$ of spinning particles with spins $s_{1}, s_{2} \cdots s_{n}$. The homogeneous and non-homogeneous parts of the amplitude can be defined as

$$
\begin{aligned}
k_{i} \cdot \mathcal{M}\left(k_{1}, k_{2} \cdots, k_{n}\right)_{\mathbf{h}} & =0 \\
k_{i} \cdot \mathcal{M}\left(k_{1}, k_{2} \cdots, k_{n}\right)_{\mathbf{n h}} & =\text { WT identity }
\end{aligned}
$$

As an example, let us consider the three graviton $d S_{4}$ amplitude. It has three contributions. Two parity-even contributions come from $\mathcal{W}^{3}$ and the Einstein gravity part, and a parityodd contribution comes from $\mathcal{W}^{2} \widetilde{\mathcal{W}}$. It can be easily checked that the contributions from $\mathcal{W}^{3}$ and $\mathcal{W}^{2} \widetilde{\mathcal{W}}$ are homogeneous whereas the Einstein gravity part is non-homogeneous. Let us check this explicitly. The contribution from $\mathcal{W}^{3}$ is given by [21]

$$
\begin{aligned}
\mathcal{M}_{\mathcal{W}^{3}} & =F(1,2) F(2,3) F(3,1) \\
F(i, j) & =\left(\epsilon_{i} \cdot k_{j} \epsilon_{j} \cdot k_{i}-\epsilon_{i} \cdot \epsilon_{j} k_{i} \cdot k_{j}\right)
\end{aligned}
$$


where $\epsilon_{i}$ are transverse polarization tensors. To analyse (4.18) we have to replace one of the polarization tensors with the momentum

$$
\epsilon_{i} \rightarrow k_{i}: \mathcal{M}_{\mathcal{W}^{3}} \rightarrow 0
$$

which implies that the $\mathcal{W}^{3}$ contribution is homogeneous. It is easy to check that a similar conclusion holds for $\mathcal{W}^{2} \widetilde{\mathcal{W}}$. Let us now consider the contribution from Einstein gravity (EG)

$$
\mathcal{M}_{E G}=\left(k_{2} \cdot \epsilon_{1} \epsilon_{2} \cdot \epsilon_{3}+\text { cyclic }\right)^{2}
$$

The analogue of (4.18) is

$$
\epsilon_{i} \rightarrow k_{i}: \mathcal{M}_{E G}=\left(k_{2} \cdot k_{1} \epsilon_{2} \cdot \epsilon_{3}+\text { cyclic }\right)\left(k_{2} \cdot \epsilon_{1} \epsilon_{2} \cdot \epsilon_{3}+\text { cyclic }\right) \neq 0 .
$$

Thus this contribution is non-homogeneous. It is interesting to point out that in gravity, there exists a natural distinction between the homogeneous and non-homogeneous parts. In CFT, the homogeneous and non-homogeneous parts come together as we saw in the case of the free bosonic and free fermionic theories (4.14).

A useful gauge where amplitudes take a simple form is given by

$$
k^{\mu}=(k, \vec{k}) \quad \epsilon^{\mu}=(0, \vec{\epsilon})
$$

In this gauge the $\mathcal{M}_{\mathcal{W}^{3}}$ structure reduces to

$$
\begin{aligned}
\mathcal{M}_{\mathcal{W}^{3}}= & {\left[\vec{\epsilon}_{1} \cdot \vec{\epsilon}_{2} E\left(E-2 k_{3}\right)+2 \vec{\epsilon}_{1} \cdot \vec{k}_{2} \vec{\epsilon}_{2} \cdot \vec{k}_{1}\right]\left[\vec{\epsilon}_{2} \cdot \vec{\epsilon}_{3} E\left(E-2 k_{1}\right)+2 \vec{\epsilon}_{2} \cdot \vec{k}_{3} \vec{\epsilon}_{3} \cdot \vec{k}_{2}\right] } \\
& {\left[\vec{\epsilon}_{3} \cdot \vec{\epsilon}_{1} E\left(E-2 k_{2}\right)+2 \vec{\epsilon}_{3} \cdot \vec{k}_{1} \vec{\epsilon}_{1} \cdot \vec{k}_{2}\right] }
\end{aligned}
$$

The other interaction which gives rise to non-homogeneous contributions is the YangMills term ${ }^{5}$ whereas terms such as $F^{3}, F^{2} \widetilde{F}, \phi F^{2}$ and $\phi F \widetilde{F}$ contribute to homogeneous amplitudes.

\subsection{Summary of 3-point functions for exactly conserved currents}

Let us consider correlation functions comprising conserved currents with spins $s_{1}, s_{2}, s_{3}$ such that they satisfy triangle inequality

$$
s_{i} \leq s_{j}+s_{k}
$$

where $i, j, k$ can be any of $1,2,3$. The most general three-point function inside the triangle can be written as the sum of parity-even free boson and free fermion contributions and a parity-odd contribution

$$
\left\langle J_{s_{1}} J_{s_{2}} J_{s_{3}}\right\rangle=c_{b}\left\langle J_{s_{1}} J_{s_{2}} J_{s_{3}}\right\rangle_{\mathrm{FB}}+c_{f}\left\langle J_{s_{1}} J_{s_{2}} J_{s_{3}}\right\rangle_{\mathrm{FF}}+c_{\mathrm{odd}}\left\langle J_{s_{1}} J_{s_{2}} J_{s_{3}}\right\rangle_{\mathrm{odd}}
$$

\footnotetext{
${ }^{5}$ In the terminology of the recent paper [51], interactions with minimal coupling such as Einstein gravity and Yang-Mills give rise to non-homogeneous contributions whereas interactions with non-minimal coupling such as $\mathcal{M}_{\mathcal{W}^{3}}$ contribute to the homogeneous part.
} 
which can also be written as

$$
\left\langle J_{s_{1}} J_{s_{2}} J_{s_{3}}\right\rangle=c_{\mathbf{h}}\left\langle J_{s_{1}} J_{s_{2}} J_{s_{3}}\right\rangle_{\mathbf{h}}+c_{\mathbf{n h}}\left\langle J_{s_{1}} J_{s_{2}} J_{s_{3}}\right\rangle_{\mathbf{n h}}+c_{\text {odd }}\left\langle J_{s_{1}} J_{s_{2}} J_{s_{3}}\right\rangle_{\text {odd }}
$$

where we used from (4.6)

$$
\begin{aligned}
& \left\langle J_{s_{1}} J_{s_{2}} J_{s_{3}}\right\rangle_{\mathrm{FB}}=\left\langle J_{s_{1}} J_{s_{2}} J_{s_{3}}\right\rangle_{\mathbf{n h}}+\left\langle J_{s_{1}} J_{s_{2}} J_{s_{3}}\right\rangle_{\mathbf{h}} \\
& \left\langle J_{s_{1}} J_{s_{2}} J_{s_{3}}\right\rangle_{\mathrm{FF}}=\left\langle J_{s_{1}} J_{s_{2}} J_{s_{3}}\right\rangle_{\mathbf{n h}}-\left\langle J_{s_{1}} J_{s_{2}} J_{s_{3}}\right\rangle_{\mathbf{h}}
\end{aligned}
$$

Correlation functions involving a scalar operator of scaling dimension $\Delta$ can be written as

$$
\begin{aligned}
\left\langle J_{s} J_{s} O_{\Delta}\right\rangle & =c_{e}\left\langle J_{s} J_{s} O_{\Delta}\right\rangle_{\mathbf{h}, \text { even }}+c_{o}\left\langle J_{s} J_{s} O_{\Delta}\right\rangle_{\mathbf{h}, \text { odd }} \\
\left\langle J_{s} O_{\Delta} O_{\Delta}\right\rangle & =\left\langle J_{s} O_{\Delta} O_{\Delta}\right\rangle_{\mathbf{n h}, \text { even }}
\end{aligned}
$$

Note that there is no homogeneous contribution to $\left\langle J_{s} O_{\Delta} O_{\Delta}\right\rangle$ and no non-homogeneous ${ }^{6}$ contribution to $\left\langle J_{s} J_{s} O_{\Delta}\right\rangle$.

Let us now consider the case when the spins violate triangle inequality, i.e. say

$$
s_{1}>s_{2}+s_{3}
$$

where for simplicity we have assumed $s_{1}>s_{2}$ and $s_{2} \geq s_{3}$. In such cases when the currents are exactly conserved there is no parity-odd contribution to the correlation function and the only contributions are non-homogeneous [52]

$$
\begin{aligned}
\left\langle J_{s_{1}} J_{s_{2}} J_{s_{3}}\right\rangle & =\left\langle J_{s_{1}} J_{s_{2}} J_{s_{3}}\right\rangle_{\mathrm{nh}} \\
& =c_{b}\left\langle J_{s_{1}} J_{s_{2}} J_{s_{3}}\right\rangle_{\mathrm{FB}}+c_{f}\left\langle J_{s_{1}} J_{s_{2}} J_{s_{3}}\right\rangle_{\mathrm{FF}}
\end{aligned}
$$

Thus we see that the correlator is parity-even and gets only non-homogeneous contributions.

One can also show that for correlation functions with one scalar operator and two exactly conserved currents with unequal spins $s_{1}$ and $s_{2}$

$$
\begin{aligned}
& \left\langle J_{s_{1}} J_{s_{2}} O_{\Delta}\right\rangle \neq 0 \text { for } \mathrm{s}_{1} \neq \mathrm{s}_{2} \text { for } \Delta=1,2 \\
& \left\langle J_{s_{1}} J_{s_{2}} O_{1}\right\rangle=\left\langle J_{s_{1}} J_{s_{2}} O_{1}\right\rangle_{\text {even,nh }} \\
& \left\langle J_{s_{1}} J_{s_{2}} O_{2}\right\rangle=\left\langle J_{s_{1}} J_{s_{2}} O_{2}\right\rangle_{\text {odd,nh }} .
\end{aligned}
$$

\section{Relating parity-even and odd correlation function for exactly con- served currents}

In this section we work out the relation between the parity-even and the parity-odd parts of correlation functions in spinor-helicity variables, momentum space and in position space. The homogeneous part of correlation functions in spinor-helicity variables was discussed in [41]. In position space it is given by (4.16). In [41], momentum space expressions were also written down. However, to construct a map from parity-even to parity-odd, it is convenient to rewrite the expressions in a slightly different way which makes the map manifest. We will start our analysis using spinor-helicity variables.

\footnotetext{
${ }^{6}$ There can be a contact term that can arise in $\left\langle J_{s} J_{s} O\right\rangle$. However such terms can be suitably redefined to zero.
} 


\subsection{Relation in spinor-helicity variables}

Consider a general correlator of the form $\left\langle J_{s_{1}} J_{s_{2}} J_{s_{3}}\right\rangle$ where $s_{1} \geq s_{2} \geq s_{3}$ and $s_{2}+s_{3}>s_{1}$. In spinor-helicity variables, the homogeneous part of the correlator is given by

$$
\left\langle J_{s_{1}}^{-} J_{s_{2}}^{-} J_{s_{3}}^{-}\right\rangle_{\mathbf{h}}=\left(c_{\text {even }}+i c_{\text {odd }}\right) \frac{k_{1}^{s_{1}-1} k_{2}^{s_{2}-1} k_{3}^{s_{3}-1}}{E^{s_{1}+s_{2}+s_{3}}}\langle 12\rangle^{s_{1}+s_{2}-s_{3}}\langle 23\rangle^{s_{2}+s_{3}-s_{1}}\langle 31\rangle^{s_{1}+s_{3}-s_{2}}
$$

The only other non-zero contribution to the homogeneous piece comes from the +++ helicity component which can be obtained by complex conjugating (5.1). The other helicity components have only non-homogeneous contribution [41]. It is interesting to note that the parity-even and the parity-odd parts of the homogeneous part of the correlation function are the same up to factors of $i$. To avoid clutter, we introduce the following notation

$$
\begin{aligned}
a & \equiv \frac{1}{2}\left(s_{1}+s_{2}-s_{3}\right), \quad b \equiv \frac{1}{2}\left(s_{2}+s_{3}-s_{1}\right), \quad c \equiv \frac{1}{2}\left(s_{1}+s_{3}-s_{2}\right) \\
S & \equiv s_{1}+s_{2}+s_{3}
\end{aligned}
$$

\subsection{Relation in momentum space}

The homogeneous part of any 3-point function of the form $\left\langle J_{s_{1}} J_{s_{2}} J_{s_{3}}\right\rangle$ can be written in terms of a finite number of building blocks as was observed in [41]. In [41] we had introduced

$$
\begin{aligned}
Q_{12}= & \frac{1}{E^{2}}\left[2\left(\vec{z}_{1} \cdot \vec{k}_{2}\right)\left(\vec{z}_{2} \cdot \vec{k}_{1}\right)+E\left(E-2 k_{3}\right) \vec{z}_{1} \cdot \vec{z}_{2}\right] \\
S_{12}=\frac{2}{E^{2}}\left[k_{1} \epsilon^{k_{2} z_{1} z_{2}}-k_{2} \epsilon^{k_{1} z_{1} z_{2}}\right] & \\
P_{123}=\frac{1}{E^{3}} & {\left[2\left(\vec{z}_{1} \cdot \vec{k}_{2}\right)\left(\vec{z}_{2} \cdot \vec{k}_{3}\right)\left(\vec{z}_{3} \cdot \vec{k}_{1}\right)+E\left(k_{3}\left(\vec{z}_{1} \cdot \vec{z}_{2}\right)\left(\vec{z}_{3} \cdot \vec{k}_{1}\right)+\text { cyclic }\right)\right] } \\
R_{123}=\frac{1}{E^{3}} & {\left[\left\{\left(\vec{k}_{1} \cdot \vec{z}_{3}\right)\left(\epsilon^{k_{3} z_{1} z_{2}} k_{1}-\epsilon^{k_{1} z_{1} z_{2}} k_{3}\right)+\left(\vec{k}_{3} \cdot \vec{z}_{2}\right)\left(\epsilon^{k_{1} z_{1} z_{3}} k_{2}-\epsilon^{k_{2} z_{1} z_{3}} k_{1}\right)\right.\right.} \\
& \left.\left.\quad-\left(\vec{z}_{2} \cdot \vec{z}_{3}\right) \epsilon^{k_{1} k_{2} z_{1}} E+\frac{k_{1}}{2} \epsilon^{z_{1} z_{2} z_{3}} E\left(E-2 k_{1}\right)\right\}+ \text { cyclic perm }\right]
\end{aligned}
$$

Let us split correlators into two families, one satisfying $s_{1}+s_{2}+s_{3}=$ even and the other satisfying $s_{1}+s_{2}+s_{3}=$ odd.

$s_{1}+s_{2}+s_{3}=\mathbf{2 n}(n \in \mathbb{Z})$. The parity-even and the parity-odd homogeneous pieces of correlators involving a scalar operator are given by

$$
\begin{aligned}
\left\langle J_{s} J_{s} O_{2}\right\rangle_{\text {even }} & =\frac{b_{12}^{2 s-2}}{E^{2 s-2}} Q_{12}\left(z_{1} \cdot z_{2}\right)^{s-1} \\
\left\langle J_{s} J_{s} O_{2}\right\rangle_{\text {odd }} & =\frac{b_{12}^{2 s-2}}{E^{2 s-2}} S_{12}\left(z_{1} \cdot z_{2}\right)^{s-1} .
\end{aligned}
$$

Correlation functions involving all spinning operators are given by

$$
\begin{aligned}
\left\langle J_{s_{1}} J_{s_{2}} J_{s_{3}}\right\rangle_{\text {even }} & =\frac{k_{1}^{s_{1}-1} k_{2}^{s_{2}-1} k_{3}^{s_{3}-1}}{E^{S-4}}\left(z_{1} \cdot z_{3}\right)^{c-1}\left(z_{2} \cdot z_{3}\right)^{b-1}\left(z_{1} \cdot z_{2}\right)^{a} Q_{13} Q_{23} \\
\left\langle J_{s_{1}} J_{s_{2}} J_{s_{3}}\right\rangle_{\text {odd }} & =\frac{k_{1}^{s_{1}-1} k_{2}^{s_{2}-1} k_{3}^{s_{3}-1}}{E^{S-4}}\left(z_{1} \cdot z_{3}\right)^{c-1}\left(z_{2} \cdot z_{3}\right)^{b-1}\left(z_{1} \cdot z_{2}\right)^{a-1} \epsilon^{k_{1} z_{1} z_{2}} Q_{13} Q_{23} \\
& =\frac{k_{1}^{s_{1}-1} k_{2}^{s_{2}-1} k_{3}^{s_{3}-1}}{E^{S-4}}\left(z_{1} \cdot z_{3}\right)^{c-1}\left(z_{2} \cdot z_{3}\right)^{b-1}\left(z_{1} \cdot z_{2}\right)^{a} S_{13} Q_{23}
\end{aligned}
$$


Let us note that the results presented in (5.4) and (5.5) are significantly simpler than what appears in [41]. To reach these results we have made repeated use of degeneracy and Schouten identities. The details are worked out in appendix A. Results in (5.4) and (5.5) can be symmetrized appropriately. However, it can be shown that all such symmetrized terms are related by degeneracy and Schouten identities to the expressions in (5.4) and (5.5).

Let us now consider the second family of correlators in which $s_{1}+s_{2}+s_{3}=$ odd.

$s_{1}+s_{2}+s_{3}=\mathbf{2 n}+\mathbf{1}(\boldsymbol{n} \in \mathbb{Z})$. When $s_{1}+s_{2}+s_{3}=$ odd for general spins $s_{1}, s_{2}, s_{3}$, the correlator can be written as

$$
\begin{aligned}
\left\langle J_{s_{1}} J_{s_{2}} J_{s_{3}}\right\rangle_{\text {even }, \mathbf{h}} & =\frac{k_{1}^{2\left(s_{1}-1\right)} k_{2}^{2\left(s_{2}-1\right)} k_{3}^{2\left(s_{3}-1\right)}}{E^{S-3}} P_{123}\left(z_{1} \cdot z_{2}\right)^{a-1 / 2}\left(z_{1} \cdot z_{3}\right)^{c-1 / 2}\left(z_{2} \cdot z_{3}\right)^{b-1 / 2} \\
\left\langle J_{s_{1}} J_{s_{2}} J_{s_{3}}\right\rangle_{\text {odd }, \mathbf{h}} & =\frac{k_{1}^{2\left(s_{1}-1\right)} k_{2}^{2\left(s_{2}-1\right)} k_{3}^{2\left(s_{3}-1\right)}}{E^{S-3}} R_{123}\left(z_{1} \cdot z_{2}\right)^{a-1 / 2}\left(z_{1} \cdot z_{3}\right)^{c-1 / 2}\left(z_{2} \cdot z_{3}\right)^{b-1 / 2}
\end{aligned}
$$

Relating parity-even to parity-odd. From their expressions in (5.3) one can check that

$$
\frac{1}{k_{1}} \epsilon^{k_{1} z_{1} z_{2}} P_{123}=-k_{1} k_{2} z_{1} \cdot z_{2} R_{123}
$$

Furthermore $R_{123}$ and $P_{123}$ are related by

$$
\frac{1}{k_{i}} \epsilon^{\mu k_{i} z_{i}} \frac{\partial}{\partial z_{i}^{\mu}} P_{123}=R_{123}
$$

We can see that (5.8) and (5.9) map parity-even correlators to parity-odd correlators. We see the following simple relation between the parity-even and the parity-odd results

$$
\frac{1}{k_{1}} \epsilon^{z_{1} z_{2} k_{1}} \frac{\partial}{\partial\left(z_{1} \cdot z_{2}\right)}: \text { even }_{\mathbf{h}} \rightarrow \text { odd }
$$

Let us note that for $s_{1} \neq 0, s_{2} \neq 0, s_{3} \neq 0$ we could have chosen any $z_{i} \cdot z_{j}$ and replaced it with either $\frac{1}{k_{i}} \epsilon_{i}^{z_{i} z_{j} k_{i}}$ or $\frac{1}{k_{j}} \epsilon^{z_{i} z_{j} k_{j}}$ and it would have given us the map (5.10). We also note that the map in (5.10) is exactly the same as the one observed at the level of two-point functions in (2.10).

Using Todorov operator one can rewrite the relation in (5.10) as

$$
\left\langle J_{s_{1}} J_{s_{2}} J_{s_{3}}\right\rangle_{\text {even, } \mathbf{h}} \mapsto \frac{1}{k_{1}} \epsilon_{\alpha}^{k_{1}\left(\mu_{1}\right.}\left\langle J_{s_{1}}^{\left.\mu_{2} \cdots \mu_{s_{1}}\right) \alpha} J_{s_{2}} J_{s_{3}}\right\rangle_{\text {even, } \mathbf{h}}
$$

This maps the parity-even homogeneous contribution in momentum space to the parity-odd contribution

$$
\left\langle J_{s_{1}} J_{s_{2}} J_{s_{3}}\right\rangle_{\text {odd }, \mathbf{h}}=\frac{1}{k_{1}} \epsilon_{\alpha}^{k_{1}\left(\mu_{1}\right.}\left\langle J_{s_{1}}^{\left.\mu_{2} \cdots \mu_{s_{1}}\right) \alpha} J_{s_{2}} J_{s_{3}}\right\rangle_{\text {even, } \mathbf{h}}
$$

One can check that this reduces to the relation one has in spinor-helicity variables. 
Summary of map. Using all the results discussed above one can show the following in general $^{7}$

$$
\begin{gathered}
\frac{1}{k_{1}} \epsilon^{\mu_{1} \alpha k_{1}}\left\langle J_{\alpha}^{\mu_{2} \cdots \mu_{s_{1}}}\left(k_{1}\right) J^{\nu_{1} \cdots \nu_{s_{2}}}\left(k_{2}\right) J^{\rho_{1} \cdots \rho_{s_{3}}}\left(k_{3}\right)\right\rangle_{\text {even, } \mathbf{h}}+\left(\mu_{1} \leftrightarrow \mu_{2}\right)+\cdots+\left(\mu_{1} \leftrightarrow \mu_{s_{1}}\right) \\
=\left\langle J^{\mu_{1} \cdots \mu_{s_{1}}}\left(k_{1}\right) J^{\nu_{1} \cdots \nu_{s_{2}}}\left(k_{2}\right) J^{\rho_{1} \cdots \rho_{s_{3}}}\left(k_{3}\right)\right\rangle_{\text {odd }} \\
-\frac{1}{k_{1}} \epsilon^{\mu_{1} \alpha k_{1}}\left\langle J_{\alpha}^{\mu_{2} \cdots \mu_{s_{1}}}\left(k_{1}\right) J^{\nu_{1} \cdots \nu_{s_{2}}}\left(k_{2}\right) J^{\rho_{1} \cdots \rho_{s_{3}}}\left(k_{3}\right)\right\rangle_{\text {odd }}+\left(\mu_{1} \leftrightarrow \mu_{2}\right)+\cdots+\left(\mu_{1} \leftrightarrow \mu_{s_{1}}\right) \\
=\left\langle J^{\mu_{1} \cdots \mu_{s_{1}}}\left(k_{1}\right) J^{\nu_{1} \cdots \nu_{s_{2}}}\left(k_{2}\right) J^{\rho_{1} \cdots \rho_{s_{3}}}\left(k_{3}\right)\right\rangle_{\text {even, } \mathbf{h}} .
\end{gathered}
$$

In the above we used the first spin for mapping. However note that the mapping works out exactly the same way for the second and the third spins as well.

\subsection{Relation in position space}

The relation between the parity-even and the parity-odd parts of the correlation function in momentum space can easily be Fourier transformed to get the relations in position space. One can define a kernel which maps the parity-even contribution to the parity-odd contribution. The inverse kernel also exists which maps the parity-odd contribution to the parity-even contribution.

Let us start with the two-point function of the spin-1 current

$$
\begin{aligned}
\left\langle J_{\mu}(x) J_{\nu}(y)\right\rangle_{\text {even }} & =\left(-\delta_{\mu \nu}+\frac{2(x-y)_{\mu}(x-y)_{\nu}}{(x-y)^{2}}\right) \frac{1}{(x-y)^{4}} \\
\left\langle J_{\mu}(x) J_{\nu}(y)\right\rangle_{\text {odd }} & =\epsilon_{\mu \nu \alpha} \partial^{\alpha} \delta^{3}(x-y) .
\end{aligned}
$$

The parity-odd piece is a contact term. This is true for the parity-odd part of the two-point function of any spin- $s$ conserved current.

For the spin- 1 current it is easy to check that the parity-odd and the parity-even parts are related as

$$
\left\langle J_{\mu}(x) J_{\nu}(y)\right\rangle_{\text {odd }}=\epsilon_{\mu \sigma \alpha} \int \frac{d^{3} x_{1}}{\left|x-x_{1}\right|^{2}} \partial_{x_{1}}^{\sigma}\left\langle J^{\alpha}\left(x_{1}\right) J_{\nu}(y)\right\rangle_{\text {even }}
$$

where $\partial_{x_{1}}^{\sigma}=\frac{\partial}{\partial x_{1, \sigma}}$.

Three-point function. We now turn our attention to three-point functions. A Fourier transform of the relation in momentum space gives

$$
\left\langle J^{\mu_{1} \mu_{2} \cdots \mu_{s_{1}}}\left(y_{1}\right) J_{s_{2}}\left(y_{2}\right) J_{s_{3}}\left(y_{3}\right)\right\rangle_{\text {odd }}=\epsilon_{\sigma \alpha}^{\left(\mu_{1}\right.} \int \frac{d^{3} x_{1}}{\left|y_{1}-x_{1}\right|^{2}} \partial_{x_{1}}^{\sigma}\left\langle J^{\left.\alpha \mu_{2} \cdots \mu_{s_{1}}\right)}\left(x_{1}\right) J_{s_{2}}\left(y_{2}\right) J_{s_{3}}\left(y_{3}\right)\right\rangle_{\mathrm{even}, \mathbf{h}}
$$

\footnotetext{
${ }^{7}$ Let us now consider the above relation in the light-cone coordinates $d s^{2}=2 d x^{+} d x^{-}+d x_{3}^{2}$. Let us also consider momenta that have only non-zero component along the third direction. The relation then takes the following form
}

$$
\operatorname{sgn}\left(k_{1,3}\right)\left\langle J^{--\cdots-}\left(k_{1}\right) J^{-\cdots-}\left(k_{2}\right) J^{-\cdots-}\left(k_{3}\right)\right\rangle_{\text {even } \mathbf{h}}=\left\langle J^{-\cdots-}\left(k_{1}\right) J^{-\cdots-}\left(k_{2}\right) J^{-\cdots-}\left(k_{3}\right)\right\rangle_{\text {odd }} .
$$


where we have suppressed indices of the second and third operators. There are other useful representations as well such as

$$
\epsilon_{\sigma \alpha}^{\left(\mu_{1}\right.} \partial_{y_{1}}^{\sigma}\left\langle J^{\left.\alpha \mu_{2} \cdots \mu_{s_{1}}\right)}\left(y_{1}\right) J_{s_{2}}\left(y_{2}\right) J_{s_{3}}\left(y_{3}\right)\right\rangle_{\text {odd }}=-\int \frac{d^{3} x_{1}}{\left|y_{1}-x_{1}\right|^{4}}\left\langle J^{\mu_{1} \mu_{2} \cdots \mu_{s_{1}}}\left(x_{1}\right) J_{s_{2}}\left(y_{2}\right) J_{s_{3}}\left(y_{3}\right)\right\rangle_{\text {even, }}
$$

It is possible to invert the relation and express the parity-even result in terms of the parity-odd result as follows

$$
\left\langle J^{\mu_{1} \mu_{2} \cdots \mu_{s_{1}}}\left(y_{1}\right) J_{s_{2}}\left(y_{2}\right) J_{s_{3}}\left(y_{3}\right)\right\rangle_{\text {even, } \mathbf{h}}=-\epsilon_{\sigma \alpha}^{\left(\mu_{1}\right.} \int \frac{d^{3} x_{1}}{\left|y_{1}-x_{1}\right|^{2}} \partial_{x_{1}}^{\sigma}\left\langle J^{\left.\alpha \mu_{2} \cdots \mu_{s_{1}}\right)}\left(x_{1}\right) J_{s_{2}}\left(y_{2}\right) J_{s_{3}}\left(y_{3}\right)\right\rangle_{\text {odd }}
$$

which can also be rewritten as

$\epsilon_{\sigma \alpha}^{\left(\mu_{1}\right.} \partial_{y_{1}}^{\sigma}\left\langle J^{\left.\alpha \mu_{2} \cdots \mu_{s_{1}}\right)}\left(y_{1}\right) J_{s_{2}}\left(y_{2}\right) J_{s_{3}}\left(y_{3}\right)\right\rangle_{\text {even, } \mathbf{h}}=\int \frac{d^{3} x_{1}}{\left|y_{1}-x_{1}\right|^{4}}\left\langle J^{\mu_{1} \mu_{2} \cdots \mu_{s_{1}}}\left(x_{1}\right) J_{s_{2}}\left(y_{2}\right) J_{s_{3}}\left(y_{3}\right)\right\rangle_{\text {odd }}$.

Using (4.12), we can symbolically write

$$
\left\langle J^{\mu_{1} \mu_{2} \cdots \mu_{s_{1}}}\left(y_{1}\right) J_{s_{2}}^{\nu_{1} \cdots \nu_{s_{2}}}\left(y_{2}\right) J_{s_{3}}^{\gamma_{1} \cdots \gamma_{s_{3}}}\left(y_{3}\right)\right\rangle_{\text {odd }}=\epsilon^{\left(\mu_{1} \sigma \alpha\right.} \int \frac{d^{3} x_{1}}{\left|y_{1}-x_{1}\right|^{2}} \partial_{x_{1}}^{\sigma} \mathcal{F}_{\mathbf{h}}{ }^{\left.\alpha \mu_{2} \cdots \mu_{s_{1}}\right) \nu_{1} \cdots \nu_{s_{2}} \gamma_{1} \cdots \gamma_{s_{3}}}
$$

Let us denote the kernel by $\mathcal{K}$. We then have

$$
\begin{aligned}
\left\langle J_{s_{1}} J_{s_{2}} J_{s_{3}}\right\rangle_{\text {odd }} & =\mathcal{K}\left[\mathcal{F}_{\mathbf{h}}\right] \\
& =\mathcal{K}\left[e^{\frac{Q_{1}+Q_{2}+Q_{3}}{2}} e^{P_{1}+P_{2}+P_{3}}\right] \\
& =\frac{1}{2} \mathcal{K}\left[\left\langle J_{s_{1}} J_{s_{2}} J_{s_{3}}\right\rangle_{\mathrm{FF}}-\left\langle J_{s_{1}} J_{s_{2}} J_{s_{3}}\right\rangle_{\mathrm{FB}}\right]
\end{aligned}
$$

We shall derive this relation in section 6 . As a concrete example one can consider $\left\langle J_{\mu} J_{\nu} O_{\Delta}\right\rangle$ given in (4.9). Using the star triangle relation, ${ }^{8}$ it is straightforward to show that

$$
\left\langle J^{\mu}\left(x_{1}\right) J^{\nu}\left(x_{2}\right) O_{\Delta}\left(x_{3}\right)\right\rangle_{\text {odd }}=\epsilon^{\mu \sigma \alpha} \int \frac{d^{3} y_{1}}{\left|y_{1}-x_{1}\right|^{2}} \partial_{x_{1}}^{\sigma}\left\langle J^{\alpha}\left(y_{1}\right) J^{\nu}\left(x_{2}\right) O_{\Delta}\left(x_{3}\right)\right\rangle_{\text {even }} .
$$

\subsection{Relation between parity-even and parity-odd three-point $d S_{4}$ amplitude}

It is known that CFT correlation functions and $d S_{4}$ amplitudes are related to each other [1]. This readily implies that the parity-even and the parity-odd parts of the $d S_{4}$ amplitudes are related just as they are in CFT correlators. To be explicit in the computations, we will consider the three-point graviton amplitude. The parity-even parts of the correlator are

\footnotetext{
${ }^{8}$ The star triangle relation is given by
}

$$
\int d^{3} x \frac{1}{\left|x-y_{1}\right|^{2 \Delta_{1}}\left|x-y_{2}\right|^{2 \Delta_{2}}\left|x-y_{3}\right|^{2 \Delta_{3}}} \propto \frac{1}{y_{12}^{3-2 \Delta_{3}} y_{13}^{3-2 \Delta_{2}} y_{23}^{3-2 \Delta_{1}}}
$$

where $\sum_{i=1}^{3} \Delta_{i}=3$. 
given by (4.19) and (4.21). The parity-odd part of the amplitude is

$$
\begin{aligned}
\mathcal{M}_{\mathcal{W}^{2} \widetilde{\mathcal{W}}}=[ & \left.2\left(\vec{z}_{1} \cdot \vec{k}_{2} \vec{z}_{2} \cdot \vec{k}_{3} \vec{z}_{3} \cdot \vec{k}_{1}\right)+E\left(\left(\vec{z}_{1} \cdot \vec{z}_{2} \vec{z}_{3} \cdot \vec{k}_{1}\right) k_{3}+\text { cyclic }\right)\right] \\
\times & {\left[\left(\vec{z}_{2} \cdot \vec{k}_{3}\right)\left(\epsilon^{k_{3} z_{1} z_{2}} k_{1}+\epsilon^{k_{1} z_{1} z_{3}}\left(E-k_{3}\right)\right)+\left(\vec{k}_{1} \cdot \vec{z}_{3}\right)\left(\epsilon^{k_{2} z_{1} z_{2}} k_{1}+\epsilon^{k_{1} z_{1} z_{2}}\left(E-k_{2}\right)\right)\right.} \\
& \left.-E\left(\vec{z}_{2} \cdot \vec{z}_{3}\right) \epsilon^{k_{1} k_{2} z_{1}}+\frac{1}{2} k_{1} E\left(E-2 k_{1}\right) \epsilon^{z_{1} z_{2} z_{3}}+\text { cyclic perm. }\right]
\end{aligned}
$$

We choose a gauge in which $k^{\mu}=\left(|\vec{k}|, \vec{k}_{i}\right)$ and $z^{\mu}=(0, \vec{z})$. It is easy to check that the contributions from $\mathcal{W}^{3}$ and $\mathcal{W}^{2} \widetilde{\mathcal{W}}$ are related to each other by

$$
\frac{1}{k_{i}} \epsilon^{\mu k_{i} z_{i}} \frac{\partial}{\partial z_{i}^{\mu}} \mathcal{M}_{W^{3}} \propto \mathcal{M}_{W^{2} \widetilde{W}}
$$

Similar relations hold between contributions from $\phi W \widetilde{W}$ and $\phi W^{2}, \phi F \widetilde{F}$ and $\phi F^{2}$, and $F^{2} \widetilde{F}$ and $F^{3}$. Similar statements can be made for interactions involving higher-spins. We also notice that there is no such relation involving the amplitude given by Einstein gravity part (4.21).

\subsection{Four-point correlator}

Very few results exist for four-point functions in momentum space [53-55]. For details on scalar four-point correlators see [56-60].

In this section we first argue abstractly that if there is a parity-even homogeneous solution to the conformal Ward identity then there also exists a parity-odd homogeneous solution. To show this let us consider the simplest example of $\langle T O O O\rangle$.

\subsection{1 $\langle\text { TOOO }\rangle_{\text {even }}$}

The ansatz for the transverse-traceless part of the parity-even correlator is given by

$$
\left\langle T^{\mu \nu}\left(k_{1}\right) O\left(k_{2}\right) O\left(k_{3}\right) O\left(k_{4}\right)\right\rangle_{\text {even }}=\Pi_{\alpha \beta}^{\mu \nu}\left(k_{1}\right)\left(A_{1} k_{2}^{\alpha} k_{2}^{\beta}+A_{2} k_{3}^{\alpha} k_{3}^{\beta}+A_{3} k_{4}^{\alpha} k_{4}^{\beta}\right)
$$

The WT identity for the correlator is [36]

$$
\begin{aligned}
k_{1 \mu}\left\langle T^{\mu \nu}\left(k_{1}\right) O\left(k_{2}\right) O\left(k_{3}\right) O\left(k_{4}\right)\right\rangle_{\mathrm{even}}= & -k_{2}^{\nu}\left\langle O\left(k_{3}+k_{3}\right) O\left(k_{3}\right) O\left(k_{4}\right)\right\rangle-k_{3}^{\nu}\left\langle O\left(k_{2}+k_{3}\right) O\left(k_{2}\right) O\left(k_{4}\right)\right\rangle \\
& -k_{4}^{\nu}\left\langle O\left(k_{2}+k_{3}\right) O\left(k_{2}\right) O\left(k_{3}\right)\right\rangle
\end{aligned}
$$

We separate the correlation function into homogeneous and non-homogeneous parts

$$
\langle T O O O\rangle=\langle T O O O\rangle_{\mathbf{h}, \text { even }}+\langle T O O O\rangle_{\mathbf{n h}, \text { even }}
$$

Let us now convert the ansatz in (5.27) to spinor-helicity variables

$$
\left\langle T^{-}\left(k_{1}\right) O\left(k_{2}\right) O\left(k_{3}\right) O\left(k_{4}\right)\right\rangle_{\text {even }}=A_{1}\langle 12\rangle^{2}\langle\overline{2} 1\rangle^{2}+A_{2}\langle 13\rangle^{2}\langle\overline{3} 1\rangle^{2}+A_{3}\langle 14\rangle^{2}\langle\overline{4} 1\rangle^{2} .
$$

The positive helicity component is obtained by complex conjugation. 
The conformal Ward identity in spinor-helicity variables is given by

$$
\widetilde{K}^{\kappa}\left\langle\frac{T^{-}}{k_{1}} O O O\right\rangle=12 \frac{z_{1}^{-\kappa}}{k_{1}^{3}} z_{(1 \mu}^{-} k_{1 \nu)}\left\langle T^{\mu \nu} O O O\right\rangle
$$

where $z_{1}^{-\kappa}=\frac{\left(\sigma^{\kappa}\right)^{\alpha \beta} \lambda_{1 \alpha} \lambda_{1 \beta}}{2 k_{1}}$. Plugging (5.29) in (5.31) we see that the homogeneous piece satisfies

$$
\widetilde{K}^{\kappa}\left\langle\frac{T^{-}}{k_{1}} O O O\right\rangle_{\mathbf{h}, \text { even }}=0
$$

In general it is not known how to solve this equation in terms of an arbitrary function of conformal cross-ratios. However, for our purpose, it is sufficient to know just the equation (5.32).

\subsection{2 $\langle\text { TOOO }\rangle_{\text {odd }}$}

Let us now write down ansatz for the parity-odd contribution. It can be written as

$$
\left\langle T^{\mu \nu}\left(k_{1}\right) O\left(k_{2}\right) O\left(k_{3}\right) O\left(k_{4}\right)\right\rangle_{\text {odd }}=\Delta_{\alpha \beta}^{\mu \nu}\left(k_{1}\right)\left(B_{1} k_{2}^{\alpha} k_{2}^{\beta}+B_{2} k_{3}^{\alpha} k_{3}^{\beta}+B_{3} k_{4}^{\alpha} k_{4}^{\beta}\right)
$$

The WT identity for the parity-odd part is trivial

$$
k_{1 \mu}\left\langle T^{\mu \nu}\left(k_{1}\right) O\left(k_{2}\right) O\left(k_{3}\right) O\left(k_{4}\right)\right\rangle_{\text {odd }}=0 .
$$

Thus we see that the parity-odd part has only homogeneous contribution

$$
\langle T O O O\rangle_{\text {odd }}=\langle T O O O\rangle_{\mathbf{h}, \text { odd }} .
$$

Transforming the ansatz (5.33) to spinor-helicity variables we obtain

$$
\left\langle T^{-} O O O\right\rangle_{\text {odd }}=B_{1}\langle 12\rangle^{2}\langle\overline{2} 1\rangle^{2}+B_{2}\langle 13\rangle^{2}\langle\overline{3} 1\rangle^{2}+B_{3}\langle 14\rangle^{2}\langle\overline{4} 1\rangle^{2}
$$

Let us note that even though the parity-odd ansatz (5.33) looks completely different from the parity-even ansatz (5.27), in spinor-helicity variables they become identical. The conformal Ward identity is again given by

$$
\widetilde{K}^{\kappa}\left\langle\frac{T^{-}}{k_{1}} O O O\right\rangle_{\mathbf{h}, \text { odd }}=0 .
$$

It is easy to show that if $\left\{A_{1}, A_{2}, A_{3}\right\}$ is a solution to (5.32), one of the solutions of (5.37) is $\left\{B_{1}, B_{2}, B_{3}\right\}$ where

$$
B_{1}=A_{1}, \quad B_{2}=A_{2}, \quad B_{3}=A_{3} .
$$

As in the case of three-point functions, we find a map between the parity-even and the parity-odd homogeneous parts of the correlator as follows

$$
\begin{aligned}
\frac{1}{k_{1}} \epsilon_{\gamma}{ }^{k_{1}(\mu}\left\langle T^{\nu) \gamma} O O O\right\rangle_{\text {even }} & =\frac{1}{k_{1}} \epsilon_{\gamma}{ }^{k_{1}(\mu} \Pi_{\alpha \beta}^{\nu) \gamma}\left(k_{1}\right)\left(A_{1} k_{2}^{\alpha} k_{2}^{\beta}+A_{2} k_{3}^{\alpha} k_{3}^{\beta}+A_{3} k_{4}^{\alpha} k_{4}^{\beta}\right) \\
& =\Delta_{\alpha \beta}^{\mu \nu}\left(k_{1}\right)\left(A_{1} k_{2}^{\alpha} k_{2}^{\beta}+A_{2} k_{3}^{\alpha} k_{3}^{\beta}+A_{3} k_{4}^{\alpha} k_{4}^{\beta}\right) \\
& =\left\langle T^{\mu \nu} O O O\right\rangle_{\text {odd }}
\end{aligned}
$$


One can easily generalize this discussion to correlation functions involving more than one spinning operator as well as to arbitrary $n$-point functions. We conclude that for an arbitrary $n$-point function, we can construct a parity-odd homogeneous solution using a parity-even homogeneous solution by doing the epsilon-transformation we discussed above.

\section{A derivation using weakly broken higher spin symmetry}

In this section we make use of weakly broken higher spin symmetry to derive relations presented in the previous sections between parity-even and parity-odd correlation functions. Until now we considered only exactly conserved currents. We will now consider theories with weakly broken higher-spin symmetry at large $N$. We show that for two-point functions of such currents the relation between even and odd correlators continues to hold. For three-point functions when the spins satisfy triangle inequality, the same relation holds between the even and odd correlators. For weakly broken higher spin currents, parity-odd contribution to correlators exist even when triangle inequality is violated. We show that the relation between even and odd continues to hold in this case.

Three-point function with slightly broken higher spin current. In theories with weakly broken higher-spin symmetry even when triangle inequality is violated (4.30), there is one parity-odd contribution in (4.26), i.e.

$$
\left\langle J_{s_{1}} J_{s_{2}} J_{s_{3}}\right\rangle_{\text {odd }} \neq 0 \quad \text { even outside the triangle. }
$$

We also have

$$
\begin{aligned}
& \left\langle J_{s_{1}} J_{s_{2}} O_{\Delta}\right\rangle \neq 0 \text { for } \mathrm{s}_{1} \neq \mathrm{s}_{2} \text { for } \Delta=1,2 \\
& \left\langle J_{s_{1}} J_{s_{2}} O_{\Delta}\right\rangle=\left\langle J_{s_{1}} J_{s_{2}} O_{\Delta}\right\rangle_{\text {even }}+\left\langle J_{s_{1}} J_{s_{2}} O_{\Delta}\right\rangle_{\text {odd }}
\end{aligned}
$$

It turns out that all the contribution to the correlator when the spins violate triangle inequality is non-homogeneous [52]. Let us also note that when the spins satisfy triangle inequality, there is no change in the structure of the correlation function.

A derivation of the parity-even-odd relation. In this section we use slightly broken higher symmetry [25] to derive the relation between the parity-even and the parity-odd part of correlation functions. ${ }^{9}$ Let us start with correlation functions of the form $\left\langle T_{\mu \nu} J_{s_{1}} J_{s_{2}}\right\rangle$ where spins $s_{1}$ and $s_{2}$ are arbitrary and the currents need not be conserved. In the following we also use the notation

$$
X_{z_{i} z_{j} z_{k}}=X_{\mu \nu \rho} z_{i}^{\mu} z_{j}^{\nu} z_{k}^{\rho}
$$

where $z_{i}$ are polarization vectors such that

$$
z_{i}^{2}=0, \quad z_{i} \cdot k_{i}=0
$$

\footnotetext{
${ }^{9}$ In this section we closely follow appendix D of [25].
} 


\section{1 $\left\langle T_{\mu \nu} J_{s_{1}} J_{s_{2}}\right\rangle$}

Let us consider the spin- 4 current $J_{4}$ that obeys the following non-conservation equation in the quasi-fermionic theory [25]

$$
\partial_{\sigma} J_{z_{1} z_{1} z_{1}}^{\sigma}=\frac{80}{7} \frac{\widetilde{\lambda}}{1+\widetilde{\lambda}^{2}}\left(\partial_{z_{1}} O T_{z_{1} z_{1}}-\frac{2}{5} O \partial_{z_{1}} T_{z_{1} z_{1}}\right)
$$

where we have used the notation $z^{\mu} \partial_{\mu}=\partial_{z}$. In (6.5), $T$ denotes the trace of the stress tensor, ${ }^{10} O$ denotes the scalar operator with scaling dimension $\Delta=2+\mathcal{O}\left(\frac{1}{N}\right)$ and $\widetilde{\lambda}$ is the coupling constant introduced in [25].

The charge associated to $J_{4}$ labelled $Q_{4}$ has the following action on $O$

$$
\left[Q_{z_{1} z_{1} z_{1}}, O\right]=\epsilon_{z_{1} a b} \partial_{a} \partial_{z_{1}} T_{z_{1}}^{b}+\partial_{z_{1}}^{3} O
$$

Let us now consider the action of $Q_{4}$ on the three-point correlator $\left\langle O J_{s_{1}} J_{s_{2}}\right\rangle$. It leads to the following higher spin equation in position space

$$
\begin{aligned}
& \left\langle\left[Q_{z_{1} z_{1} z_{1}}, O\left(x_{1}\right)\right] J_{s_{1}}\left(x_{2}\right) J_{s_{2}}\left(x_{3}\right)\right\rangle_{\mathrm{QF}}+\left\langle O\left(x_{1}\right)\left[Q_{z_{1} z_{1} z_{1}}, J_{s_{1}}\left(x_{2}\right)\right] J_{s_{2}}\left(x_{3}\right)\right\rangle_{\mathrm{QF}} \\
& \quad+\left\langle O\left(x_{1}\right) J_{s_{1}}\left(x_{2}\right)\left[Q_{z_{1} z_{1} z_{1}}, J_{s_{2}}\left(x_{3}\right)\right]\right\rangle_{\mathrm{QF}}=\int_{x}\left\langle\partial_{\sigma} J_{z_{1} z_{1} z_{1}}^{\sigma}(x) O\left(x_{1}\right) J_{s_{1}}\left(x_{2}\right) J_{s_{2}}\left(x_{3}\right)\right\rangle_{\mathrm{QF}}
\end{aligned}
$$

Upon utilizing the algebra in (6.6) and the current equation (6.5) we get

$$
\epsilon_{z_{1} a b}\left\langle\partial_{a} \partial_{z_{1}} T_{z_{1}}^{b} J_{s_{1}} J_{s_{2}}\right\rangle+\langle\text { standard terms }\rangle=16 \frac{\widetilde{\lambda}}{1+\widetilde{\lambda}^{2}} \int_{x}\left\langle O \partial_{z_{1}} T_{z_{1} z_{1}} O J_{s_{1}} J_{s_{2}}\right\rangle
$$

where by $\langle$ standard terms $\rangle$ we mean correlators with a single insertion of the scalar operator $O$. After a large $N$ factorisation we obtain the following

$$
\epsilon_{z_{1} a b}\left\langle\partial_{a} \partial_{z_{1}} T_{z_{1}}^{b} J_{s_{1}} J_{s_{2}}\right\rangle+\langle\text { standard terms }\rangle=16 \frac{\widetilde{\lambda}}{1+\widetilde{\lambda}^{2}} \int_{x}\left\langle O(x) O\left(x_{1}\right)\right\rangle\left\langle\partial_{z_{1}} T_{z_{1} z_{1}}(x) J_{s_{1}} J_{s_{2}}\right\rangle
$$

We now express the three-point functions in the quasi-fermionic theory as [25]

$$
\begin{aligned}
\langle O O\rangle_{\mathrm{QF}} & =\left(1+\widetilde{\lambda}^{2}\right)\langle O O\rangle_{\mathrm{FF}} \\
\left\langle T J_{s_{1}} J_{s_{2}}\right\rangle_{\mathrm{QF}} & =\frac{\widetilde{\lambda}^{2}}{1+\widetilde{\lambda}^{2}}\left\langle T J_{s_{1}} J_{s_{2}}\right\rangle_{\mathrm{FB}}+\frac{1}{1+\widetilde{\lambda}^{2}}\left\langle T J_{s_{1}} J_{s_{2}}\right\rangle_{\mathrm{FF}}+\frac{\widetilde{\lambda}}{1+\widetilde{\lambda}^{2}}\left\langle T J_{s_{1}} J_{s_{2}}\right\rangle_{\mathrm{odd}} \\
\left\langle J_{s_{1}} J_{s_{2}} O\right\rangle_{\mathrm{QF}} & =\left\langle J_{s_{1}} J_{s_{2}} O\right\rangle_{\mathrm{FF}}+\widetilde{\lambda}\left\langle J_{s_{1}} J_{s_{2}} O\right\rangle_{\mathrm{odd}}
\end{aligned}
$$

Let us now look at terms with the pole structure $\frac{1}{1+\widetilde{\lambda}^{2}}$. This gives us the following relation

$$
\epsilon_{z_{1} a b} \partial_{a} \partial_{z_{1}}\left(\left\langle T_{z_{1}}^{b} J_{s_{1}} J_{s_{2}}\right\rangle_{\mathrm{FF}}-\left\langle T_{z_{1}}^{b} J_{s_{1}} J_{s_{2}}\right\rangle_{\mathrm{FB}}\right)=-16 \int_{x}\left\langle\partial_{z_{1}} T_{z_{1} z_{1}}(x) J_{s_{1}} J_{s_{2}}\right\rangle_{\text {odd }}\left\langle O(x) O\left(x_{1}\right)\right\rangle
$$

\footnotetext{
${ }^{10}$ Even though the stress-tensor is traceless, it can lead to a non-trivial trace WT identity.
} 
Let us now look at terms with the pole structure $\frac{\widetilde{\lambda}}{1+\widetilde{\lambda}^{2}}$. This gives us the following relation

$$
\epsilon_{z_{1} a b} \partial^{a} \partial_{z_{1}}\left\langle T_{z_{1}}^{b} J_{s_{1}} J_{s_{2}}\right\rangle_{\text {odd }}=16 \int_{x}\left(\left\langle\partial_{z_{1}} T_{z_{1} z_{1}}(x) J_{s_{1}} J_{s_{2}}\right\rangle_{\mathrm{FF}}-\left\langle\partial_{z_{1}} T_{z_{1} z_{1}} J_{s_{1}} J_{s_{2}}\right\rangle_{\mathrm{FB}}\right)\left\langle O(x) O\left(x_{1}\right)\right\rangle
$$

Now we make use of the Todorov operator [61] along with (4.17) and the fact that the trace WT identity is also identical for the free boson and the free fermion. We obtain the following two equations from the above two

$$
\begin{aligned}
& \epsilon_{\mu a b}\left(\left\langle\partial_{a} \partial_{(\nu} T_{\rho)}^{b} J_{s_{1}} J_{s_{2}}\right\rangle_{\mathrm{FF}}-\left\langle T_{\rho)}^{b} J_{s_{1}} J_{s_{2}}\right\rangle_{\mathrm{FB}}\right)+\mu \leftrightarrow \nu+\mu \leftrightarrow \rho \\
& =-16 \int_{x}\left\langle\partial_{(\mu} T_{\nu \rho)}(x) J_{s_{1}} J_{s_{2}}\right\rangle_{\text {odd }}\left\langle O(x) O\left(x_{1}\right)\right\rangle
\end{aligned}
$$

and

$$
\begin{aligned}
\epsilon_{\mu a b}\left\langle\partial_{a} \partial_{(\nu} T_{\rho)}^{b} J_{s_{1}} J_{s_{2}}\right\rangle_{\mathrm{odd}}+\mu \leftrightarrow \nu+\mu \leftrightarrow \rho \\
\quad=16 \int_{x}\left(\left\langle\partial_{(\mu} T_{\nu \rho)}(x) J_{s_{1}} J_{s_{2}}\right\rangle_{\mathrm{FF}}-\left\langle\partial_{(\mu} T_{\nu \rho)}(x) J_{s_{1}} J_{s_{2}}\right\rangle_{\mathrm{FB}}\right)\left\langle O(x) O\left(x_{1}\right)\right\rangle
\end{aligned}
$$

In Fourier space, these relations take the following form

$$
\epsilon_{\mu k_{1} b} k_{1(\nu}\left(\left\langle T_{\rho)}^{b} J_{s_{1}} J_{s_{2}}\right\rangle_{\mathrm{FF}}-\left\langle T_{\rho)}^{b} J_{s_{1}} J_{s_{2}}\right\rangle_{\mathrm{FB}}\right)+\mu \leftrightarrow \nu+\mu \leftrightarrow \rho=k_{1} k_{1(\mu}\left\langle T_{\nu \rho)} J_{s_{1}} J_{s_{2}}\right\rangle_{\text {odd }}
$$

and

$$
\epsilon_{\mu k_{1} b} k_{1(\nu}\left\langle T_{\rho)}^{b} J_{s_{1}} J_{s_{2}}\right\rangle_{\text {odd }}+\mu \leftrightarrow \nu+\mu \leftrightarrow \rho=k_{1} k_{1(\mu}\left(\left\langle T_{\nu \rho)} J_{s_{1}} J_{s_{2}}\right\rangle_{\mathrm{FB}}-\left\langle T_{\nu \rho)} J_{s_{1}} J_{s_{2}}\right\rangle_{\mathrm{FF}}\right)
$$

We now use $\Pi_{\alpha \beta}^{\nu \rho}\left(k_{1}\right)$ to project the r.h.s. and the l.h.s. of the above equations. Using the following

$$
\begin{aligned}
\Pi_{\alpha \beta}^{\nu \rho}\left(k_{1}\right) k_{1 \nu} & =\Pi_{\alpha \beta}^{\nu \rho}\left(k_{1}\right) k_{1 \rho}=0 \\
\Pi_{\alpha \beta}^{\nu \rho}\left(k_{1}\right)\left(\left\langle T_{\nu \rho} J_{s_{1}} J_{s_{2}}\right\rangle_{\mathrm{FB}}-\left\langle T_{\nu \rho} J_{s_{1}} J_{s_{2}}\right\rangle_{\mathrm{FF}}\right) & =\left\langle T_{\nu \rho} J_{s_{1}} J_{s_{2}}\right\rangle_{\mathrm{FB}}-\left\langle T_{\nu \rho} J_{s_{1}} J_{s_{2}}\right\rangle_{\mathrm{FF}}
\end{aligned}
$$

we obtain

$$
\begin{array}{r}
k_{1 \mu} \epsilon_{\left(\nu k_{1} b\right.}\left\langle T_{\rho)}^{b} J_{s_{1}} J_{s_{2}}\right\rangle_{\mathrm{odd}}=k_{1} k_{1 \mu}\left(\left\langle T_{\nu \rho} J_{s_{1}} J_{s_{2}}\right\rangle_{\mathrm{FB}}-\left\langle T_{\nu \rho} J_{s_{1}} J_{s_{2}}\right\rangle_{\mathrm{FF}}\right) \\
\Longrightarrow \epsilon_{\left(\nu k_{1} b\right.}\left\langle T_{\rho)}^{b} J_{s_{1}} J_{s_{2}}\right\rangle_{\mathrm{odd}}=k_{1}\left(\left\langle T_{\nu \rho} J_{s_{1}} J_{s_{2}}\right\rangle_{\mathrm{FB}}-\left\langle T_{\nu \rho} J_{s_{1}} J_{s_{2}}\right\rangle_{\mathrm{FF}}\right)
\end{array}
$$

Similarly we also obtain

$$
k_{1}\left\langle T_{\nu \rho} J_{s_{1}} J_{s_{2}}\right\rangle_{\mathrm{odd}}=-\epsilon_{k_{1} b(\nu}\left(\left\langle T_{\rho)}^{b} J_{s_{1}} J_{s_{2}}\right\rangle_{\mathrm{FF}}-\left\langle T_{\rho)}^{b} J_{s_{1}} J_{s_{2}}\right\rangle_{\mathrm{FB}}\right)
$$

This relation translates to the following in position space

$$
\int \frac{1}{\left|x-x_{1}\right|^{4}}\left\langle T_{\nu \rho} J_{s_{1}} J_{s_{2}}\right\rangle_{\mathrm{odd}}=\partial_{x_{1 \mu}} \epsilon_{\mu b(\nu}\left(\left\langle T_{\rho)}^{b} J_{s_{1}} J_{s_{2}}\right\rangle_{\mathrm{FF}}-\left\langle T_{\rho)}^{b} J_{s_{1}} J_{s_{2}}\right\rangle_{\mathrm{FB}}\right)
$$

In (6.19) one can as well take the $k_{1}$ on the l.h.s. to the r.h.s. and upon converting the resulting equation to position space one obtains

$$
\left\langle T_{\nu \rho} J_{s_{1}} J_{s_{2}}\right\rangle_{\mathrm{odd}}=\int \frac{1}{\left|x-x_{1}\right|^{2}} \partial_{x_{\mu}} \epsilon_{\mu b(\nu}\left(\left\langle T_{\rho)}^{b} J_{s_{1}} J_{s_{2}}\right\rangle_{\mathrm{FF}}-\left\langle T_{\rho)}^{b} J_{s_{1}} J_{s_{2}}\right\rangle_{\mathrm{FB}}\right)
$$


This is precisely the relation between odd and even correlators that we derived in (5.21). Note that we could have argued this relation directly from (6.14) by writing down the explicit structure in position space and realizing that (6.21) indeed holds. As a simple example we could have $s_{1}=s_{2}=2$ which corresponds to the $\langle T T T\rangle$ case. Even though we have used large- $N$ techniques, here we have derived results that are perfectly valid even at finite $N$ as was discussed in previous sections.

Let us now consider the case when the spins of the operators in $\left\langle T J_{s_{1}} J_{s_{2}}\right\rangle$ are such that they violate triangle inequality, i.e. $s_{1}>s_{2}+2$. In this case the correlators that appear in the higher spin equation are purely non-homogeneous. However, the above relation between parity-odd and parity-even correlators will continue to hold. Our analysis can be easily extended to operators with higher spin. See appendix B for details.

Note that one can obtain similar relations by working in the quasi-bosonic theory.

\section{$6.2\left\langle J_{s} T O\right\rangle$}

Let us consider a correlator of the kind $\left\langle J_{s} T O\right\rangle$ such that the spins violate triangle inequality, i.e. $s>2$. We will now derive the relation between the parity-odd part of $\left\langle J_{s} T O\right\rangle$ and the same correlator in free theories where it is parity-even. To do so we consider the action of $Q_{4}$ on the three-point correlator $\left\langle J_{s} O O\right\rangle$. It leads to the following higher spin equation:

$$
\begin{aligned}
& \left\langle\left[Q_{\mu \nu \rho}, J_{s}\left(x_{1}\right)\right] O\left(x_{2}\right) O\left(x_{3}\right)\right\rangle_{\mathrm{QF}}+\left\langle J_{s}\left(x_{1}\right)\left[Q_{\mu \nu \rho}, O\left(x_{2}\right)\right] O\left(x_{3}\right)\right\rangle_{\mathrm{QF}} \\
& \quad+\left\langle J_{s}\left(x_{1}\right) O\left(x_{2}\right)\left[Q_{\mu \nu \rho}, O\left(x_{3}\right)\right]\right\rangle_{\mathrm{QF}}=\int_{x}\left\langle\partial_{\sigma} J_{\mu \nu \rho}^{\sigma}(x) J_{s}\left(x_{1}\right) O\left(x_{2}\right) O\left(x_{3}\right)\right\rangle_{\mathrm{QF}}
\end{aligned}
$$

Following similar steps as above we obtain

$$
\left\langle J_{s} T_{\nu \rho} O\right\rangle_{\mathrm{odd}}=\frac{1}{k_{2}} \epsilon_{\left(\nu k_{2} b\right.}\left\langle J_{s} T_{\rho)}^{b} O\right\rangle_{\mathrm{FF}}
$$

In position space this relation takes the form

$$
\left\langle J_{s} T_{\nu \rho} O\right\rangle_{\mathrm{odd}}=\int \frac{1}{\left|x-x_{2}\right|^{2}} \partial_{x_{\mu}} \epsilon_{(\nu \mu b}\left\langle J_{s} T_{\rho)}^{b} O\right\rangle_{\mathrm{FF}}
$$

This is precisely the relation we obtained in section 5.3. The analysis can also be generalised to $\left\langle J_{s_{1}} J_{s_{2}} O\right\rangle$ of which a special case is $\left\langle J_{s} J_{s} O\right\rangle$.

\section{$6.3\langle$ TOOO $\rangle$}

In this section we obtain the relation between the parity-odd and the parity-even parts of four-point functions. Let us consider $\langle T O O O\rangle$. To obtain this correlator we consider the action of $Q_{4}$ on the four-point function of scalar operators $\langle O O O O\rangle$. Following the steps in the previous sections and making use of the following [62] (a similar identification of the quasi-fermionic correlator has been made in the position and Mellin spaces in [53]) and [55] respectively)

$$
\langle T O O O\rangle_{\mathrm{QF}}=\left(1+\widetilde{\lambda}^{2}\right)\langle T O O O\rangle_{\mathrm{FF}}+\widetilde{\lambda}\left(1+\widetilde{\lambda}^{2}\right)\langle T O O O\rangle_{\mathrm{CB}}
$$


We note that $\langle T O O O\rangle_{\mathrm{FF}}$ is parity-odd and $\langle T O O O\rangle_{\mathrm{CB}}$ is parity-even and they are homogeneous: ${ }^{11}$

$$
k_{1}^{\mu}\left\langle T_{\mu \nu} O O O\right\rangle_{F F, C B}=0
$$

From the higher spin equations we obtain

$$
\begin{aligned}
& {\left[\epsilon_{\mu a b}\left\langle\partial_{a} \partial_{(\nu} T_{\rho)}^{b} O O O\right\rangle_{\mathrm{FF}}+(\mu \leftrightarrow \nu)+(\mu \leftrightarrow \rho)\right]+\text { permutations }} \\
& =\int_{x}\left[-16\langle O O\rangle_{\mathrm{FF}}\left\langle\partial_{(\mu} T_{\nu \rho)} O O O\right\rangle_{\mathrm{CB}}+(1 \leftrightarrow 2)+(1 \leftrightarrow 3)+(1 \leftrightarrow 4)\right]
\end{aligned}
$$

and

$$
\begin{aligned}
& {\left[\epsilon_{\mu a b}\left\langle\partial_{a} \partial_{(\nu} T_{\rho)}^{b} O O O\right\rangle_{\mathrm{CB}}+(\mu \leftrightarrow \nu)+(\mu \leftrightarrow \rho)\right]+\text { permutations }} \\
& =\int_{x}\left[16\langle O O\rangle_{\mathrm{FF}}\left\langle\partial_{(\mu} T_{\nu \rho)} O O O\right\rangle_{\mathrm{FF}}+(1 \leftrightarrow 2)+(1 \leftrightarrow 3)+(1 \leftrightarrow 4)\right]
\end{aligned}
$$

In Fourier space these equations take the following form

$$
\begin{aligned}
& {\left[\epsilon_{\mu k_{1} b} k_{1(\nu}\left\langle T_{\rho)}^{b} O O O\right\rangle_{\mathrm{FF}}+(\mu \leftrightarrow \nu)+(\mu \leftrightarrow \rho)\right]+\text { permutations }} \\
& =16\langle O O\rangle_{\mathrm{FF}} k_{1(\mu}\left\langle T_{\nu \rho)} O O O\right\rangle_{\mathrm{CB}}+(1 \leftrightarrow 2)+(1 \leftrightarrow 3)+(1 \leftrightarrow 4)
\end{aligned}
$$

and

$$
\begin{aligned}
& {\left[\epsilon_{\mu k_{1} b} k_{1(\nu}\left\langle T_{\rho)}^{b} O O O\right\rangle_{\mathrm{CB}}+(\mu \leftrightarrow \nu)+(\mu \leftrightarrow \rho)\right]+\text { permutations }} \\
& =-16\langle O O\rangle_{\mathrm{FF}} k_{1(\mu}\left\langle T_{\nu \rho)} O O O\right\rangle_{\mathrm{FF}}+(1 \leftrightarrow 2)+(1 \leftrightarrow 3)+(1 \leftrightarrow 4)
\end{aligned}
$$

Thus we obtain a relation between the parity-even and the parity-odd parts of $\langle T O O O\rangle .{ }^{12}$

\section{$7 \quad$ Summary and future directions}

In this paper we explicitly showed that for exactly conserved currents, one can relate the parity-odd part of the correlation function to the parity-even homogeneous contribution to the correlator. ${ }^{13}$ We wrote down an explicit relation between the two in position space, momentum space and in spinor-helicity variables. The fact that, for exactly conserved currents, there does not exist a parity-odd non-homogeneous contribution can be understood by a simple analysis of the WT identity. However, for weakly broken higher spin theories, when the spins violate triangle inequality there are only non-homogeneous contributions. It turns out that in such cases, a relation exists between the even and the odd non-homogeneous pieces.

\footnotetext{
${ }^{11}$ The Ward identity is proportional to the 3-point $\langle O O O\rangle$ which can be set to zero for $O$ with scaling dimension 2 .

${ }^{12}$ This relation is weaker than the one we discussed in (6.30) because this holds true only with the permutations taken into account. In position space similar relations were explicitly understood in [53].

${ }^{13}$ There is no analogue of parity-odd non-homogeneous correlator which can be obtained from a parityeven non-homogeneous correlator.
} 
We explicitly wrote down a relation between the parity-even and the parity-odd parts of amplitudes in one higher dimension. We found that in four dimensions parity-even interactions arising from non-minimal coupling map to parity-odd interactions arising from nonminimal coupling. As expected gravity naturally splits up the correlation into homogeneous and non-homogeneous part [21]. We also briefly discussed the case of four-point functions.

It would be interesting to generalize our results in the following ways. Firstly, it would be interesting to verify the relation between the parity-even and the parity-odd four-point homogeneous correlation functions. It would be interesting to check if similar relations hold for the non-homogeneous parts of four-point functions. One easy way would be to see if the parity-even and the parity-odd WT identities could be mapped to each other in spinor-helicity variables. It would also be interesting to verify if similar relations exist between the parity-even and the parity-odd homogeneous parts of 4 photon and 4 graviton amplitudes [63].

Another interesting direction would be to explore how the relation between even and odd correlators comes about in perturbation theory when we compute correlation functions in Chern-Simons matter theories [45, 46, 54, 64]. The parity-odd contribution comes from odd loop orders whereas parity-even contribution comes from even loop orders. This implies that the relation between the even and odd parts of correlators gives a relation between the even and odd loop order calculations. The parity-odd contribution leads to anyonic nature exhibited by Chern-Simons matter theories [47, 65, 66]. A related story is that of flux attachment $[4,5]$. It would be interesting to explore these aspects of Chern-Simons matter theories in the light of the relation between the parity-odd and the parity-even correlators that we obtained in this paper. It would be interesting to find out if similar relation holds at finite temperature. This would lead to a relation between viscosity, conductivity and Hall viscosity and Hall conductivity computed in [3, 67].

\section{Acknowledgments}

The work of SJ and RRJ is supported by the Ramanujan Fellowship. We acknowledge our debt to the people of India for their steady support of research in basic sciences. We thank S. D. Chowdhury, L. Janagal, S. Sinha and E. Skvortsov for useful discussions and correspondence. We thank A. Mehta and A. Suresh for collaboration at an early stage of this work.

\section{A Three-point function in momentum space}

In this appendix we give details of the three-point functions used in the main text. For our purpose, it is sufficient to concentrate on the homogeneous part of the correlation function. The homogeneous part of any 3-point function of the form $\left\langle J_{s_{1}} J_{s_{2}} J_{s_{3}}\right\rangle$ can be written in 
terms of a finite number of building blocks which are given below [41].

$$
\begin{aligned}
& Q_{12}= \frac{1}{E^{2}}\left[2\left(\vec{z}_{1} \cdot \vec{k}_{2}\right)\left(\vec{z}_{2} \cdot \vec{k}_{1}\right)+E\left(E-2 k_{3}\right) \vec{z}_{1} \cdot \vec{z}_{2}\right] \\
& S_{12}= \frac{2}{E^{2}}\left[k_{1} \epsilon^{k_{2} z_{1} z_{2}}-k_{2} \epsilon^{k_{1} z_{1} z_{2}}\right] \\
& P_{123}= \frac{1}{E^{3}}\left[2\left(\vec{z}_{1} \cdot \vec{k}_{2}\right)\left(\vec{z}_{2} \cdot \vec{k}_{3}\right)\left(\vec{z}_{3} \cdot \vec{k}_{1}\right)+E\left(k_{3}\left(\vec{z}_{1} \cdot \vec{z}_{2}\right)\left(\vec{z}_{3} \cdot \vec{k}_{1}\right)+\text { cyclic }\right)\right] \\
& R_{123}=\frac{1}{E^{3}}\left[\left\{\left(\vec{k}_{1} \cdot \vec{z}_{3}\right)\left(\epsilon^{k_{3} z_{1} z_{2}} k_{1}-\epsilon^{k_{1} z_{1} z_{2}} k_{3}\right)+\left(\vec{k}_{3} \cdot \vec{z}_{2}\right)\left(\epsilon^{k_{1} z_{1} z_{3}} k_{2}-\epsilon^{k_{2} z_{1} z_{3}} k_{1}\right)\right.\right. \\
&\left.\left.\quad-\left(\vec{z}_{2} \cdot \vec{z}_{3}\right) \epsilon^{k_{1} k_{2} z_{1}} E+\frac{k_{1}}{2} \epsilon^{z_{1} z_{2} z_{3}} E\left(E-2 k_{1}\right)\right\}+ \text { cyclic perm }\right]
\end{aligned}
$$

$s_{1}+s_{2}+s_{3}=2 n(n \in \mathbb{Z})$. It was shown in [41], for this class of correlators we only require $Q_{i j}$ and $S_{i j}$. Let us consider $\left\langle J_{s_{1}} J_{s_{2}} J_{s_{3}}\right\rangle$ such that $s_{1} \geq s_{2} \geq s_{3}, s_{1} \leq s_{2}+s_{3}$. For this case we have

$$
\begin{aligned}
\left\langle J_{s_{1}} J_{s_{2}} J_{s_{3}}\right\rangle_{\text {even }}= & k_{1}^{s_{1}-1} k_{2}^{s_{2}-1} k_{3}^{s_{3}-1} Q_{12}^{\frac{1}{2}\left(s_{1}+s_{2}-s_{3}\right)} Q_{23}^{\frac{1}{2}\left(s_{2}+s_{3}-s_{1}\right)} Q_{13}^{\frac{1}{2}\left(s_{1}+s_{3}-s_{2}\right)} \\
\left\langle J_{s_{1}} J_{s_{2}} J_{s_{3}}\right\rangle_{\text {odd }}= & k_{1}^{s_{1}-1} k_{2}^{s_{2}-1} k_{3}^{s_{3}-1} S_{12} Q_{12}^{\frac{1}{2}\left(s_{1}+s_{2}-s_{3}-2\right)} Q_{23}^{\frac{1}{2}\left(s_{2}+s_{3}-s_{1}\right)} Q_{13}^{\frac{1}{2}\left(s_{1}+s_{3}-s_{2}\right)} \\
& + \text { cyclic perm. }
\end{aligned}
$$

For the correlator with scalar operators we have

$$
\begin{aligned}
\left\langle J_{s} J_{s} O_{2}\right\rangle_{\text {even, } \mathbf{h}} & =b_{12}^{s-1} Q_{12}^{s} \\
\left\langle J_{s} J_{s} O_{2}\right\rangle_{\text {odd, } \mathbf{h}} & =b_{12}^{s-1} S_{12} Q_{12}^{s-1}
\end{aligned}
$$

where $b_{i j}=k_{i} k_{j}$ and $c_{123}=k_{1} k_{2} k_{3}$. For scalar operator with $\Delta=1$ we just need to do a shadow transform to (A.6). For generic scalar operator dimension $\Delta$ can also be obtained easily. Now we use following interesting identities

$$
\begin{aligned}
\frac{1}{4} E^{2} Q_{i j}^{2} & =k_{i} k_{j} z_{i} \cdot z_{j} Q_{i j} \\
\frac{1}{4} E^{2} Q_{i j} S_{i j} & =k_{i} k_{j} z_{i} \cdot z_{j} S_{i j}=-k_{j} \epsilon^{k_{i} z_{i} z_{j}} Q_{i j}=k_{i} \epsilon^{k_{j} z_{i} z_{j}} Q_{i j} .
\end{aligned}
$$

We can easily check that

$$
\begin{aligned}
\frac{1}{4} E^{2} Q_{i j}^{2}-Q_{i j} k_{i} k_{j}\left(z_{i} \cdot z_{j}\right) & =\frac{1}{E^{2}}\left(\left(k_{2} \cdot z_{1}\right)^{2}\left(k_{3} \cdot z_{2}\right)^{2}+2\left(k_{1} \cdot k_{2}\right)\left(k_{2} \cdot z_{1}\right)\left(k_{3} \cdot z_{2}\right)\left(z_{1} \cdot z_{2}\right)-\frac{J^{2}}{4}\left(z_{1} \cdot z_{2}\right)^{2}\right) \\
& =0
\end{aligned}
$$

where $J^{2}=4\left(k_{1}^{2} k_{2}^{2}-\left(k_{1} \cdot k_{2}\right)^{2}\right.$. The last line of (A.9) is zero because r.h.s. is exactly degeneracy factor [33]. Let us emphasize the fact that

$$
\begin{aligned}
& \frac{1}{4} E^{2} Q_{i j}^{2}-k_{i} k_{j} z_{i} \cdot z_{j} Q_{i j}=0 \\
& \Longrightarrow \frac{1}{4} E^{2} Q_{i j}=k_{i} k_{j}\left(z_{i} \cdot z_{j}\right)
\end{aligned}
$$


This is because the way the degeneracy works is only with $Q_{i j}^{2}$. Using this identity we can rewrite (A.5) as follows

$$
\begin{aligned}
\left\langle J_{s_{1}} J_{s_{2}} J_{s_{3}}\right\rangle_{\text {even }}= & \frac{k_{1}^{2 s_{1}-3} k_{2}^{2 s_{2}-3} k_{3}^{2 s_{3}-3}}{E^{s_{1}+s_{2}+s_{3}-6}} Q_{12} Q_{13} Q_{23}\left(z_{1} \cdot z_{2}\right)^{\frac{s_{1}+s_{2}-s_{3}-2}{2}}\left(z_{2} \cdot z_{3}\right)^{\frac{-s_{1}+s_{2}+s_{3}-2}{2}}\left(z_{1} \cdot z_{3}\right)^{\frac{s_{1}-s_{2}+s_{3}-2}{2}} \\
\left\langle J_{s_{1}} J_{s_{2}} J_{s_{3}}\right\rangle_{\text {odd }}= & \frac{k_{1}^{2 s_{1}-3} k_{2}^{2 s_{2}-3} k_{3}^{2 s_{3}-3}}{E^{s_{1}+s_{2}+s_{3}-6}} S_{12} Q_{13} Q_{23}\left(z_{1} \cdot z_{2}\right)^{\frac{s_{1}+s_{2}-s_{3}-2}{2}}\left(z_{2} \cdot z_{3}\right)^{\frac{-s_{1}+s_{2}+s_{3}-2}{2}}\left(z_{1} \cdot z_{3}\right)^{\frac{s_{1}-s_{2}+s_{3}-2}{2}} \\
& +\operatorname{cyclic}
\end{aligned}
$$

Remarkably we can use the following identity

$$
\begin{aligned}
& \frac{1}{4} E^{2} Q_{12} Q_{13} Q_{23}=k_{2} k_{3} z_{2} \cdot z_{3} Q_{12} Q_{13}=k_{1} k_{3} z_{1} \cdot z_{3} Q_{12} Q_{23}=k_{1} k_{2} z_{1} \cdot z_{2} Q_{13} Q_{23} \\
& \frac{1}{4} E^{2} S_{12} Q_{13} Q_{23}=k_{2} k_{3} z_{2} \cdot z_{3} S_{12} Q_{13}=k_{1} k_{3} z_{1} \cdot z_{3} S_{12} Q_{23}
\end{aligned}
$$

This turns (A.11) into

$$
\begin{aligned}
& \left\langle J_{s_{1}} J_{s_{2}} J_{s_{3}}\right\rangle_{\text {even }}=\frac{k_{1}^{2 s_{1}-2} k_{2}^{2 s_{2}-3} k_{3}^{2 s_{3}-2}}{E^{s_{1}+s_{2}+s_{3}-4}}\left(z_{1} \cdot z_{3}\right)^{\frac{s_{1}-s_{2}+s_{3}}{2}}\left(z_{2} \cdot z_{3}\right)^{\frac{-s_{1}+s_{2}+s_{3}-2}{2}}\left(z_{1} \cdot z_{2}\right)^{\frac{s_{1}+s_{2}-s_{3}-2}{2}} Q_{12} Q_{23} \\
& \left\langle J_{s_{1}} J_{s_{2}} J_{s_{3}}\right\rangle_{\text {odd }}=\frac{k_{1}^{2 s_{1}-2} k_{2}^{2 s_{2}-3} k_{3}^{2 s_{3}-2}}{E^{s_{1}+s_{2}+s_{3}-4}}\left(z_{1} \cdot z_{3}\right)^{\frac{s_{1}-s_{2}+s_{3}}{2}}\left(z_{2} \cdot z_{3}\right)^{\frac{-s_{1}+s_{2}+s_{3}-2}{2}}\left(z_{1} \cdot z_{2}\right)^{\frac{s_{1}+s_{2}-s_{3}-2}{2}} S_{12} Q_{23}
\end{aligned}
$$

One can symmetrize above in 1,2,3 however they are just related by degeneracy. ${ }^{14}$ One can easily check (A.13) to be correct by going to spinor-helicity variables and matching it with (5.1). For parity-odd case there exists another useful representation. For this we use following identities

$$
\begin{aligned}
-\frac{1}{4} E^{2} S_{12} Q_{13} Q_{23} & =k_{2} \epsilon^{k_{1} z_{1} z_{2}} Q_{23} Q_{13}=k_{1} \epsilon^{k_{2} z_{1} z_{2}} Q_{23} Q_{13} \\
& =k_{2} \epsilon^{k_{3} z_{2} z_{3}} Q_{12} Q_{13}=k_{3} \epsilon^{k_{2} z_{2} z_{3}} Q_{12} Q_{13} \\
& =k_{3} \epsilon^{k_{1} z_{1} z_{3}} Q_{12} Q_{23}=k_{1} \epsilon^{k_{3} z_{1} z_{3}} Q_{12} Q_{23}
\end{aligned}
$$

Using this identities we can write the parity-odd result as follows

$$
\begin{aligned}
\left\langle J_{s_{1}} J_{s_{2}} J_{s_{3}}\right\rangle_{\text {odd }} & =\frac{k_{1}^{2 s_{1}-3} k_{2}^{2 s_{2}-2} k_{3}^{2 s_{3}-3}}{E^{s_{1}+s_{2}+s_{3}-4}}\left(z_{1} \cdot z_{3} \frac{\frac{s_{1}-s_{2}+s_{3}-2}{2}}{2}\left(z_{2} \cdot z_{3}\right)^{\frac{-s_{1}+s_{2}+s_{3}-2}{2}}\left(z_{1} \cdot z_{2}\right)^{\frac{s_{1}+s_{2}-s_{3}-2}{2}} \epsilon^{k_{1} z_{1} z_{2}} Q_{13} Q_{23}\right. \\
& =\frac{k_{1}^{2 s_{1}-3} k_{2}^{2 s_{2}-3} k_{3}^{2 s_{3}-2}}{E^{s_{1}+s_{2}+s_{3}-4}}\left(z_{1} \cdot z_{3}\right)^{\frac{s_{1}-s_{2}+s_{3}-2}{2}}\left(z_{2} \cdot z_{3}\right)^{\frac{-s_{1}+s_{2}+s_{3}-2}{2}}\left(z_{1} \cdot z_{2}\right)^{\frac{s_{1}+s_{2}-s_{3}-2}{2}} \epsilon^{k_{2} z_{2} z_{3}} Q_{13} Q_{12} \\
& =\frac{k_{1}^{2 s_{1}-2} k_{2}^{2 s_{2}-3} k_{3}^{2 s_{3}-3}}{E^{s_{1}+s_{2}+s_{3}-4}}\left(z_{1} \cdot z_{3}\right)^{\frac{s_{1}-s_{2}+s_{3}-2}{2}}\left(z_{2} \cdot z_{3}\right)^{\frac{-s_{1}+s_{2}+s_{3}-2}{2}}\left(z_{1} \cdot z_{2}\right)^{\frac{s_{1}+s_{2}-s_{3}-2}{2}} \epsilon^{k_{3} z_{1} z_{3}} Q_{12} Q_{23} .
\end{aligned}
$$

\footnotetext{
${ }^{14}$ There is a simple interpretation of the formula in (A.13). The factors $Q_{i j}$ and $S_{i j}$ are exactly the same as $\left\langle J J O_{2}\right\rangle_{\text {even }}$ and $\left\langle J J O_{2}\right\rangle_{\text {odd }}$ respectively. In spinor-helicity variables $Q^{-+}=Q^{+-}=S^{-+}=S^{+-}=0$ and the only non-zero components are ++ and -- . Since we want only the --- and +++ components of the homogeneous piece to be non-zero, we require at least two factors of $Q$ or one factor $Q$ and one factor of $S$. This will ensure that any mixed helicity component is zero. We can then appropriately multiply $z_{i} \cdot z_{j}$ to account for the spin.
} 
We can also write the odd part as

$$
\begin{aligned}
\left\langle J_{s_{1}} J_{s_{2}} J_{s_{3}}\right\rangle_{\text {odd }} & =\frac{k_{1}^{2 s_{1}-2} k_{2}^{2 s_{2}-3} k_{3}^{2 s_{3}-3}}{E^{s_{1}+s_{2}+s_{3}-4}}\left(z_{1} \cdot z_{3} \frac{\frac{s_{1}-s_{2}+s_{3}-2}{2}}{2}\left(z_{2} \cdot z_{3}\right)^{\frac{-s_{1}+s_{2}+s_{3}-2}{2}}\left(z_{1} \cdot z_{2}\right)^{\frac{s_{1}+s_{2}-s_{3}-2}{2}} \epsilon^{k_{2} z_{1} z_{2}} Q_{13} Q_{23}\right. \\
& =\frac{k_{1}^{2 s_{1}-3} k_{2}^{2 s_{2}-2} k_{3}^{2 s_{3}-3}}{E^{s_{1}+s_{2}+s_{3}-4}}\left(z_{1} \cdot z_{3}\right)^{\frac{s_{1}-s_{2}+s_{3}-2}{2}}\left(z_{2} \cdot z_{3}\right)^{\frac{-s_{1}+s_{2}+s_{3}-2}{2}}\left(z_{1} \cdot z_{2}\right)^{\frac{s_{1}+s_{2}-s_{3}-2}{2}} \epsilon^{k_{3} z_{2} z_{3}} Q_{13} Q_{12} \\
& =\frac{k_{1}^{2 s_{1}-3} k_{2}^{2 s_{2}-3} k_{3}^{2 s_{3}-2}}{E^{s_{1}+s_{2}+s_{3}-4}}\left(z_{1} \cdot z_{3}\right)^{\frac{s_{1}-s_{2}+s_{3}-2}{2}}\left(z_{2} \cdot z_{3}\right)^{\frac{-s_{1}+s_{2}+s_{3}-2}{2}}\left(z_{1} \cdot z_{2}\right)^{\frac{s_{1}+s_{2}-s_{3}-2}{2}} \epsilon^{k_{1} z_{1} z_{3}} Q_{12} Q_{23} .
\end{aligned}
$$

For the case say $s_{1}=s, s_{2}=s, s_{3}=0$ we can write (A.6) as

$$
\begin{aligned}
\left\langle J_{s} J_{s} O_{2}\right\rangle_{\text {even }} & =\frac{b_{12}^{2 s-2}}{E^{2 s-2}} Q_{12}\left(z_{1} \cdot z_{2}\right)^{s-1} \\
\left\langle J_{s} J_{s} O_{2}\right\rangle_{\text {odd }} & =\frac{b_{12}^{2 s-2}}{E^{2 s-2}} S_{12}\left(z_{1} \cdot z_{2}\right)^{s-1} \\
& =-\frac{b_{12}^{2 s-2}}{E^{2 s-2}} \frac{\epsilon^{k_{1} z_{1} z_{2}} Q_{12}}{k_{1}}\left(z_{1} \cdot z_{2}\right)^{s-2} \\
& =\frac{b_{12}^{2 s-2}}{E^{2 s-2}} \frac{\epsilon^{k_{2} z_{1} z_{2}} Q_{12}}{k_{2}}\left(z_{1} \cdot z_{2}\right)^{s-2}
\end{aligned}
$$

where we have used (A.8). As a concrete check let us consider simple example of $\langle T T O\rangle$. In [48], it was shown that

$$
\langle T T O\rangle_{\text {even,there }}=\frac{k_{1} k_{2}}{E^{4}}\left(2\left(k_{2} \cdot z_{1}\right)\left(k_{3} \cdot z_{2}\right)-\left(z_{1} \cdot z_{2}\right) E\left(E-2 k_{3}\right)\right)^{2}
$$

Now using (A.17) we get

$$
\langle T T O\rangle_{\text {even,here }}=\frac{k_{1} k_{2}}{E^{4}}\left(2\left(k_{2} \cdot z_{1}\right)\left(k_{3} \cdot z_{2}\right)-\left(z_{1} \cdot z_{2}\right) E\left(E-2 k_{3}\right)\right)\left(\left(z_{1} \cdot z_{2}\right) k_{1} k_{2}\right)
$$

If we consider the difference between the two expressions after accounting for a relative constant of $\frac{1}{4}$, we get

$$
\begin{aligned}
\langle T T O\rangle_{\text {even,here }}-\frac{1}{4}\langle T T O\rangle_{\text {even,there }} & =\left(k_{2} \cdot z_{1}\right)^{2}\left(k_{3} \cdot z_{2}\right)^{2}+2\left(k_{1} \cdot k_{2}\right)\left(k_{2} \cdot z_{1}\right)\left(k_{3} \cdot z_{2}\right)\left(z_{1} \cdot z_{2}\right)-\frac{J^{2}}{4}\left(z_{1} \cdot z_{2}\right)^{2} \\
& =0
\end{aligned}
$$

where we have used (A.9).

Let us take another example for $\langle T T T\rangle_{\text {odd }}$ illustration. In [41] we derived

$$
\langle T T T\rangle_{\text {odd, there }}=\frac{k_{1} k_{2} k_{3}}{E^{6}} S_{12} Q_{23} Q_{13} .
$$

Using representation (A.15) or (A.16) we have

$$
\langle T T T\rangle_{\text {odd, here }}=\frac{k_{1} k_{2} k_{3}}{E^{2}} \epsilon^{k_{1} z_{1} z_{3}} k_{3} Q_{12} Q_{23}
$$

It is easy to check using Schouten identity or spinor-helicity variables that the two expressions are the same up to overall numbers. More precisely one can show

$$
\langle T T T\rangle_{\text {odd,here }}+2\langle T T T\rangle_{\text {odd,there }}=0 .
$$


Relating parity-even and parity-odd. Now we have all the ingredient to relate parityeven and parity-odd results. Let us write the results in way which makes it evident

$$
\begin{aligned}
\left\langle J_{s} J_{s} O_{2}\right\rangle_{\text {even }} & =\frac{b_{12}^{2 s-2}}{E^{2 s-2}} Q_{12}\left(z_{1} \cdot z_{2}\right)^{s-1} \\
\left\langle J_{s} J_{s} O_{2}\right\rangle_{\text {odd }} & =\frac{b_{12}^{2 s-2}}{E^{2 s-2}} Q_{12} \frac{\epsilon^{k_{2} z_{1} z_{2}}}{k_{2}}\left(z_{1} \cdot z_{2}\right)^{s-2} \\
\left\langle J_{s_{1}} J_{s_{2}} J_{s_{3}}\right\rangle_{\text {even }} & =\frac{k_{1}^{2 s_{1}-2} k_{2}^{2 s_{2}-2} k_{3}^{2 s_{3}-3}}{E^{s_{1}+s_{2}+s_{3}-4}}\left(z_{1} \cdot z_{3}\right)^{\frac{s_{1}-s_{2}+s_{3}-2}{2}}\left(z_{2} \cdot z_{3}\right)^{\frac{-s_{1}+s_{2}+s_{3}-2}{2}}\left(z_{1} \cdot z_{2}\right)^{\frac{s_{1}+s_{2}-s_{3}}{2}} Q_{13} Q_{23} \\
\left\langle J_{s_{1}} J_{s_{2}} J_{s_{3}}\right\rangle_{\text {odd }} & =\frac{k_{1}^{2 s_{1}-3} k_{2}^{2 s_{2}-2} k_{3}^{2 s_{3}-3}}{E^{s_{1}+s_{2}+s_{3}-4}}\left(z_{1} \cdot z_{3}\right)^{\frac{s_{1}-s_{2}+s_{3}-2}{2}}\left(z_{2} \cdot z_{3}\right)^{\frac{-s_{1}+s_{2}+s_{3}-2}{2}}\left(z_{1} \cdot z_{2}\right)^{\frac{s_{1}+s_{2}-s_{3}-2}{2}} \epsilon^{k_{1} z_{1} z_{2}} Q_{13} Q_{23}
\end{aligned}
$$

We see simple relation between parity-even and parity-odd results

$$
\frac{1}{k_{1}} \epsilon^{z_{1} z_{2} k_{1}} \frac{\partial}{\partial\left(z_{1} \cdot z_{2}\right)}: \text { even }_{\mathbf{h}} \rightarrow \text { Odd }
$$

Let us note that for $s_{1} \neq 0, s_{2} \neq 0, s_{3} \neq 0$ we could have chosen any $z_{i} \cdot z_{j}$ and replaced it with either $\frac{1}{k_{i}} \epsilon^{z_{i} z_{j} k_{i}}$ or with $\frac{1}{k_{j}} \epsilon^{z_{i} z_{j} k_{j}}$ it would have given us the map (5.10). Also note that the map in (5.10) is exactly same as the map observed at the level of two-point function (2.10).

$\boldsymbol{s}_{\mathbf{1}}+\boldsymbol{s}_{\mathbf{2}}+\boldsymbol{s}_{\mathbf{3}}=\mathbf{2} \boldsymbol{n}+\mathbf{1}(\boldsymbol{n} \in \mathbb{Z})$. For this case, we require $P_{123}$ and $R_{123}$ as well. For example, when $s$ is odd, we have

$$
\begin{aligned}
\left\langle J_{s} J_{s} J_{s}\right\rangle_{\text {even, } \mathbf{h}} & =c_{123}^{s-1} P_{123}^{s} \\
\left\langle J_{s} J_{s} J_{s}\right\rangle_{\text {odd }, \mathbf{h}} & =c_{123}^{s-1} R_{123} P_{123}^{s-1}
\end{aligned}
$$

where $c_{123}=k_{1} k_{2} k_{3}$. Now using the fact that

$$
\begin{aligned}
9 P_{123}^{2} & =R_{123}^{2}=\frac{16}{9} Q_{12} Q_{13} Q_{23} \\
\frac{1}{4} E^{2} P_{123} Q_{i j} & =k_{i} k_{j} z_{i} \cdot z_{j} P_{123}, \quad \frac{1}{4} E^{2} R_{123} Q_{i j}=k_{i} k_{j} z_{i} \cdot z_{j} R_{123}
\end{aligned}
$$

we get

$$
\begin{aligned}
\left\langle J_{s} J_{s} J_{s}\right\rangle_{\text {even }, \mathbf{h}} & =\frac{c_{123}^{2(s-1)}}{E^{3 s-3}} P_{123}\left(z_{1} \cdot z_{2} z_{1} \cdot z_{3} z_{2} \cdot z_{3}\right)^{\frac{s-1}{2}} \\
\left\langle J_{s} J_{s} J_{s}\right\rangle_{\text {odd }, \mathbf{h}} & =\frac{c_{123}^{2(s-1)}}{E^{3 s-3}} R_{123}\left(z_{1} \cdot z_{2} z_{1} \cdot z_{3} z_{2} \cdot z_{3}\right)^{\frac{s-1}{2}}
\end{aligned}
$$

Finally when $s_{1}+s_{2}+s_{3}=$ odd for general spins, it is easy to check that the answer is

$$
\begin{aligned}
\left\langle J_{s_{1}} J_{s_{2}} J_{s_{3}}\right\rangle_{\text {even }, \mathbf{h}} & =\frac{k_{1}^{2\left(s_{1}-1\right)} k_{2}^{2\left(s_{2}-1\right)} k_{3}^{2\left(s_{3}-1\right)}}{E^{s_{1}+s_{2}+s_{3}-3}} P_{123}\left(z_{1} \cdot z_{2}\right)^{a}\left(z_{1} \cdot z_{3}\right)^{b}\left(z_{2} \cdot z_{3}\right)^{c} \\
\left\langle J_{s_{1}} J_{s_{2}} J_{s_{3}}\right\rangle_{\text {odd }, \mathbf{h}} & =\frac{k_{1}^{2\left(s_{1}-1\right)} k_{2}^{2\left(s_{2}-1\right)} k_{3}^{2\left(s_{3}-1\right)}}{E^{s_{1}+s_{2}+s_{3}-3}} R_{123}\left(z_{1} \cdot z_{2}\right)^{a}\left(z_{1} \cdot z_{3}\right)^{b}\left(z_{2} \cdot z_{3}\right)^{c}
\end{aligned}
$$


where

$$
\begin{aligned}
a & =\frac{1}{2}\left(s_{1}+s_{2}-s_{3}-1\right) \\
b & =\frac{1}{2}\left(s_{1}+s_{3}-s_{2}-1\right) \\
c & =\frac{1}{2}\left(s_{2}+s_{3}-s_{1}-1\right)
\end{aligned}
$$

One can also check that

$$
\frac{1}{k_{1}} \epsilon^{k_{1} z_{1} z_{2}} P_{123}=-k_{1} k_{2} z_{1} \cdot z_{2} R_{123}
$$

Further more $R_{123}$ and $P_{123}$ are related by

$$
\frac{1}{k_{i}} \epsilon^{\mu k_{i} z_{i}} \frac{\partial}{\partial z_{i}^{\mu}} P_{123}=R_{123}
$$

We see that (5.8) or (5.9) maps parity-even correlator to parity-odd correlator.

\section{B Higher-spin correlators}

Let us consider the correlator $\left\langle J_{4} J_{s_{1}} J_{s_{2}}\right\rangle$. We consider the action of the charge $Q_{6}$ associated to the spin-6 current $J_{6}$ on the correlator $\left\langle O J_{s_{1}} J_{s_{2}}\right\rangle$. To do so we make use of the following algebra

$$
\left[Q_{----}, O\right]=\epsilon_{-a b} \partial_{a} \partial_{-} J_{b---}+\epsilon_{-a b} \partial_{a} \partial_{-}^{3} T_{b-}+\partial_{-}^{5} O
$$

and the current equation

$$
\partial_{\sigma} J_{-----}^{\sigma}=16 \frac{\tilde{\lambda}}{1+\widetilde{\lambda}^{2}}\left[\partial_{-} J_{----} O+\partial_{-} T_{--} \partial_{-}^{2} O\right]
$$

The higher spin equation that arises from the action of $Q_{6}$ on $\left\langle O J_{s_{1}} J_{s_{2}}\right\rangle$ is

$$
\begin{aligned}
& \left\langle\left[Q_{6}, O\left(x_{1}\right)\right] J_{s_{1}}\left(x_{2}\right) J_{s_{2}}\left(x_{3}\right)\right\rangle_{\mathrm{QF}}+\left\langle O\left(x_{1}\right)\left[Q_{6}, J_{s_{1}}\left(x_{2}\right)\right] J_{s_{2}}\left(x_{3}\right)\right\rangle_{\mathrm{QF}} \\
& \quad+\left\langle O\left(x_{1}\right) J_{s_{1}}\left(x_{2}\right)\left[Q_{6}, J_{s_{2}}\left(x_{3}\right)\right]\right\rangle_{\mathrm{QF}}=\int_{x}\left\langle\partial_{\sigma} J_{-----}^{\sigma}(x) O\left(x_{1}\right) J_{s_{1}}\left(x_{2}\right) J_{s_{2}}\left(x_{3}\right)\right\rangle_{\mathrm{QF}}
\end{aligned}
$$

This leads to

$$
\begin{gathered}
\epsilon_{-a b} \partial_{a} \partial_{-}\left\langle J_{b---} J_{s_{1}} J_{s_{2}}\right\rangle+\epsilon_{-a b} \partial_{a} \partial_{-}^{3}\left\langle T_{b-} J_{s_{1}} J_{s_{2}}\right\rangle+\text { standard terms } \\
=\frac{\widetilde{\lambda}}{1+\widetilde{\lambda}^{2}} \int_{x}\langle O O\rangle\left\langle\partial_{-} J_{----} J_{s_{1}} J_{s_{2}}\right\rangle+\left\langle\partial_{-}^{2} O O\right\rangle\left\langle\partial_{-} T_{--} J_{s_{1}} J_{s_{2}}\right\rangle
\end{gathered}
$$

One can make use of the higher spin equation (6.9) to get rid of correlators involving the stress-tensor from the above equation. This leaves us with the following

$$
\epsilon_{-a b} \partial_{a} \partial_{-}\left\langle J_{b---} J_{s_{1}} J_{s_{2}}\right\rangle+\text { standard terms }=\frac{\tilde{\lambda}}{1+\widetilde{\lambda}^{2}} \int_{x}\left\langle\partial_{-}^{2} O O\right\rangle\left\langle\partial_{-} T_{--} J_{s_{1}} J_{s_{2}}\right\rangle
$$

We now express the three-point correlator

$$
\left\langle J_{4} J_{s_{1}} J_{s_{2}}\right\rangle_{\mathrm{QF}}=\frac{\widetilde{\lambda}^{2}}{1+\widetilde{\lambda}^{2}}\left\langle J_{4} J_{s_{1}} J_{s_{2}}\right\rangle_{\mathrm{FB}}+\frac{1}{1+\widetilde{\lambda}^{2}}\left\langle J_{4} J_{s_{1}} J_{s_{2}}\right\rangle_{\mathrm{FF}}+\frac{\widetilde{\lambda}}{1+\widetilde{\lambda}^{2}}\left\langle J_{4} J_{s_{1}} J_{s_{2}}\right\rangle_{\text {odd }}
$$


At $\mathcal{O}\left(\frac{1}{1+\widetilde{\lambda}^{2}}\right)$ we obtain

$$
\epsilon_{-a b}\left(\left\langle\partial_{a} \partial_{-} J_{b---} J_{s_{1}} J_{s_{2}}\right\rangle_{\mathrm{FB}}-\left\langle\partial_{a} \partial_{-} J_{b---} J_{s_{1}} J_{s_{2}}\right\rangle_{\mathrm{FF}}\right)=\int_{x}\left\langle O(x) O\left(x_{1}\right)\right\rangle\left\langle\partial_{-} J_{----}(x) J_{s_{1}} J_{s_{2}}\right\rangle_{\mathrm{odd}}
$$

$\operatorname{At} \mathcal{O}\left(\frac{\widetilde{\lambda}}{1+\widetilde{\lambda}^{2}}\right)$ we obtain

$$
\epsilon_{-a b}\left\langle\partial_{a} \partial_{-} J_{b---} J_{s_{1}} J_{s_{2}}\right\rangle_{\mathrm{odd}}=\int_{x}\left\langle O(x) O\left(x_{1}\right)\right\rangle\left(\left\langle\partial_{-} J_{----}(x) J_{s_{1}} J_{s_{2}}\right\rangle_{\mathrm{FF}}-\left\langle\partial_{-} J_{----}(x) J_{s_{1}} J_{s_{2}}\right\rangle_{\mathrm{FB}}\right)
$$

In Fourier space one obtains the following equations

$$
\left\langle J_{----} J_{s_{1}} J_{s_{2}}\right\rangle_{\mathrm{odd}}=k_{1} \epsilon_{-k_{1} b}\left(\left\langle J_{b---} J_{s_{1}} J_{s_{2}}\right\rangle_{\mathrm{FB}}-\left\langle J_{b---} J_{s_{1}} J_{s_{2}}\right\rangle_{\mathrm{FF}}\right)
$$

and

$$
k_{1} \epsilon_{-k_{1} b}\left\langle J_{b---} J_{s_{1}} J_{s_{2}}\right\rangle_{\mathrm{odd}}=\left\langle J_{----} J_{s_{1}} J_{s_{2}}\right\rangle_{\mathrm{FF}}-\left\langle J_{----} J_{s_{1}} J_{s_{2}}\right\rangle_{\mathrm{FB}}
$$

Open Access. This article is distributed under the terms of the Creative Commons Attribution License (CC-BY 4.0), which permits any use, distribution and reproduction in any medium, provided the original author(s) and source are credited.

\section{References}

[1] J.M. Maldacena and G.L. Pimentel, On graviton non-Gaussianities during inflation, JHEP 09 (2011) 045 [arXiv: 1104.2846] [INSPIRE].

[2] I. Mata, S. Raju and S. Trivedi, CMB from CFT, JHEP 07 (2013) 015 [arXiv:1211.5482] [INSPIRE].

[3] M. Geracie, M. Goykhman and D.T. Son, Dense Chern-Simons matter with fermions at large N, JHEP 04 (2016) 103 [arXiv:1511.04772] [INSPIRE].

[4] A. Karch and D. Tong, Particle-vortex duality from 3d bosonization, Phys. Rev. X 6 (2016) 031043 [arXiv: 1606. 01893] [INSPIRE].

[5] N. Seiberg, T. Senthil, C. Wang and E. Witten, A duality web in $2+1$ dimensions and condensed matter physics, Annals Phys. 374 (2016) 395 [arXiv: 1606. 01989] [INSPIRE].

[6] J. Murugan and H. Nastase, Particle-vortex duality in topological insulators and superconductors, JHEP 05 (2017) 159 [arXiv: 1606.01912] [INSPIRE].

[7] S. Minwalla, A. Mishra and N. Prabhakar, Fermi seas from Bose condensates in Chern-Simons matter theories and a bosonic exclusion principle, JHEP 11 (2020) 171 [arXiv: 2008.00024] [INSPIRE].

[8] O. Aharony, O. Bergman and D.L. Jafferis, Fractional M2-branes, JHEP 11 (2008) 043 [arXiv: 0807.4924] [INSPIRE].

[9] O. Aharony, O. Bergman, D.L. Jafferis and J. Maldacena, $N=6$ superconformal Chern-Simons-matter theories, M2-branes and their gravity duals, JHEP 10 (2008) 091 [arXiv:0806.1218] [INSPIRE].

[10] M.A. Vasiliev, More on equations of motion for interacting massless fields of all spins in (3+1)-dimensions, Phys. Lett. B 285 (1992) 225 [INSPIRE]. 
[11] M.A. Vasiliev, Higher spin gauge theories in four-dimensions, three-dimensions, and two-dimensions, Int. J. Mod. Phys. D 5 (1996) 763 [hep-th/9611024] [INSPIRE].

[12] M.A. Vasiliev, Higher spin gauge theories: star product and AdS space, hep-th/9910096 [INSPIRE].

[13] M.A. Vasiliev, Nonlinear equations for symmetric massless higher spin fields in $(A) d S_{d}$, Phys. Lett. B 567 (2003) 139 [hep-th/0304049] [INSPIRE].

[14] I.R. Klebanov and A.M. Polyakov, AdS dual of the critical $O(N)$ vector model, Phys. Lett. $B$ 550 (2002) 213 [hep-th/0210114] [INSPIRE].

[15] E. Sezgin and P. Sundell, Massless higher spins and holography, Nucl. Phys. B 644 (2002) 303 [Erratum ibid. 660 (2003) 403] [hep-th/0205131] [INSPIRE].

[16] S. Giombi and X. Yin, Higher spin gauge theory and holography: the three-point functions, JHEP 09 (2010) 115 [arXiv:0912.3462] [InSPIRE].

[17] S. Giombi, S. Minwalla, S. Prakash, S.P. Trivedi, S.R. Wadia and X. Yin, Chern-Simons theory with vector fermion matter, Eur. Phys. J. C 72 (2012) 2112 [arXiv:1110.4386] [INSPIRE].

[18] O. Aharony, G. Gur-Ari and R. Yacoby, $d=3$ bosonic vector models coupled to Chern-Simons gauge theories, JHEP 03 (2012) 037 [arXiv:1110.4382] [INSPIRE].

[19] S. Jain, S.P. Trivedi, S.R. Wadia and S. Yokoyama, Supersymmetric Chern-Simons theories with vector matter, JHEP 10 (2012) 194 [arXiv:1207.4750] [INSPIRE].

[20] G. Gur-Ari and R. Yacoby, Three dimensional bosonization from supersymmetry, JHEP 11 (2015) 013 [arXiv: 1507.04378] [inSPIRE].

[21] S. Giombi, S. Prakash and X. Yin, A note on CFT correlators in three dimensions, JHEP 07 (2013) 105 [arXiv: 1104.4317] [INSPIRE].

[22] M.S. Costa, J. Penedones, D. Poland and S. Rychkov, Spinning conformal correlators, JHEP 11 (2011) 071 [arXiv:1107.3554] [INSPIRE].

[23] M.S. Costa, J. Penedones, D. Poland and S. Rychkov, Spinning conformal blocks, JHEP 11 (2011) 154 [arXiv: 1109.6321] [INSPIRE].

[24] J. Maldacena and A. Zhiboedov, Constraining conformal field theories with a higher spin symmetry, J. Phys. A 46 (2013) 214011 [arXiv:1112.1016] [INSPIRE].

[25] J. Maldacena and A. Zhiboedov, Constraining conformal field theories with a slightly broken higher spin symmetry, Class. Quant. Grav. 30 (2013) 104003 [arXiv:1204.3882] [INSPIRE].

[26] S. Giombi, V. Gurucharan, V. Kirilin, S. Prakash and E. Skvortsov, On the higher-spin spectrum in large $N$ Chern-Simons vector models, JHEP 01 (2017) 058 [arXiv:1610.08472] [INSPIRE].

[27] H. Osborn and A.C. Petkou, Implications of conformal invariance in field theories for general dimensions, Annals Phys. 231 (1994) 311 [hep-th/9307010] [INSPIRE].

[28] J. Erdmenger and H. Osborn, Conserved currents and the energy momentum tensor in conformally invariant theories for general dimensions, Nucl. Phys. B 483 (1997) 431 [hep-th/9605009] [INSPIRE].

[29] C. Corianò, L. Delle Rose, E. Mottola and M. Serino, Solving the conformal constraints for scalar operators in momentum space and the evaluation of Feynman's master integrals, JHEP 07 (2013) 011 [arXiv: 1304.6944] [INSPIRE].

[30] A. Bzowski, P. McFadden and K. Skenderis, Implications of conformal invariance in momentum space, JHEP 03 (2014) 111 [arXiv:1304.7760] [INSPIRE]. 
[31] A. Bzowski, P. McFadden and K. Skenderis, Renormalised 3-point functions of stress tensors and conserved currents in CFT, JHEP 11 (2018) 153 [arXiv:1711.09105] [INSPIRE].

[32] H. Isono, T. Noumi and G. Shiu, Momentum space approach to crossing symmetric CFT correlators, JHEP 07 (2018) 136 [arXiv:1805.11107] [INSPIRE].

[33] A. Bzowski, P. McFadden and K. Skenderis, Renormalised CFT 3-point functions of scalars, currents and stress tensors, JHEP 11 (2018) 159 [arXiv:1805.12100] [INSPIRE].

[34] H. Isono, T. Noumi and T. Takeuchi, Momentum space conformal three-point functions of conserved currents and a general spinning operator, JHEP 05 (2019) 057 [arXiv: 1903.01110] [INSPIRE].

[35] H. Isono, T. Noumi and G. Shiu, Momentum space approach to crossing symmetric CFT correlators. Part II. General spacetime dimension, JHEP 10 (2019) 183 [arXiv:1908. 04572] [INSPIRE].

[36] C. Corianò, M.M. Maglio and D. Theofilopoulos, Four-point functions in momentum space: conformal Ward identities in the scalar/tensor case, Eur. Phys. J. C 80 (2020) 540 [arXiv: 1912.01907] [INSPIRE].

[37] C. Corianò and M.M. Maglio, Conformal field theory in momentum space and anomaly actions in gravity: the analysis of three- and four-point functions, arXiv:2005.06873 [INSPIRE].

[38] S. Jain, R.R. John and V. Malvimat, Momentum space spinning correlators and higher spin equations in three dimensions, JHEP 11 (2020) 049 [arXiv:2005.07212] [INSPIRE].

[39] S. Jain, R.R. John, A. Mehta, A.A. Nizami and A. Suresh, Momentum space parity-odd CFT 3-point functions, arXiv:2101.11635 [INSPIRE].

[40] S. Caron-Huot and Y.-Z. Li, Helicity basis for three-dimensional conformal field theory, $\operatorname{arXiv}: 2102.08160$ [INSPIRE].

[41] S. Jain, R.R. John, A. Mehta, A.A. Nizami and A. Suresh, Higher spin 3-point functions in 3d CFT using spinor-helicity variables, JHEP 09 (2021) 041 [arXiv:2106.00016] [INSPIRE].

[42] B. Nagaraj and D. Ponomarev, Spinor-helicity formalism for massless fields in AdS 4 , Phys. Rev. Lett. 122 (2019) 101602 [arXiv:1811.08438] [INSPIRE].

[43] R.R. Metsaev, Light-cone gauge cubic interaction vertices for massless fields in AdS $S_{4}$, Nucl. Phys. B 936 (2018) 320 [arXiv:1807.07542] [InSPIRE].

[44] E. Skvortsov, Light-front bootstrap for Chern-Simons matter theories, JHEP 06 (2019) 058 [arXiv: 1811.12333] [INSPIRE].

[45] O. Aharony, G. Gur-Ari and R. Yacoby, Correlation functions of large $N$ Chern-Simons-matter theories and bosonization in three dimensions, JHEP 12 (2012) 028 [arXiv:1207.4593] [INSPIRE].

[46] G. Gur-Ari and R. Yacoby, Correlators of large $N$ fermionic Chern-Simons vector models, JHEP 02 (2013) 150 [arXiv:1211.1866] [INSPIRE].

[47] Y. Gandhi, S. Jain and R.R. John, Anyonic correlation functions in Chern-Simons matter theories, arXiv:2106.09043 [INSPIRE].

[48] S. Jain, R.R. John, A. Mehta, A.A. Nizami and A. Suresh, Double copy structure of parity-violating CFT correlators, JHEP 07 (2021) 033 [arXiv:2104.12803] [INSPIRE].

[49] D. Baumann, C. Duaso Pueyo, A. Joyce, H. Lee and G.L. Pimentel, The cosmological bootstrap: spinning correlators from symmetries and factorization, SciPost Phys. 11 (2021) 071 [arXiv : 2005. 04234] [INSPIRE]. 
[50] A. Zhiboedov, A note on three-point functions of conserved currents, arXiv:1206.6370 [INSPIRE].

[51] D. Baumann, W.-M. Chen, C. Duaso Pueyo, A. Joyce, H. Lee and G.L. Pimentel, Linking the singularities of cosmological correlators, arXiv:2106.05294 [INSPIRE].

[52] S. Jain, R.R. John, A. Mehta and D.K. S, Constraining momentum space CFT correlators with consistent position space OPE limit and the collider bound, arXiv:2111.08024 [INSPIRE].

[53] Z. Li, Bootstrapping conformal four-point correlators with slightly broken higher spin symmetry and 3D bosonization, JHEP 10 (2020) 007 [arXiv:1906.05834] [INSPIRE].

[54] R.R. Kalloor, Four-point functions in large N Chern-Simons fermionic theories, JHEP 10 (2020) 028 [arXiv: 1910.14617] [inSPIRE].

[55] J.A. Silva, Four point functions in CFT's with slightly broken higher spin symmetry, JHEP 05 (2021) 097 [arXiv:2103.00275] [INSPIRE].

[56] A. Bedhotiya and S. Prakash, A test of bosonization at the level of four-point functions in Chern-Simons vector models, JHEP 12 (2015) 032 [arXiv:1506.05412] [INSPIRE].

[57] A. Bzowski, P. McFadden and K. Skenderis, Conformal n-point functions in momentum space, Phys. Rev. Lett. 124 (2020) 131602 [arXiv:1910.10162] [INSPIRE].

[58] A. Bzowski, P. McFadden and K. Skenderis, Conformal correlators as simplex integrals in momentum space, JHEP 01 (2021) 192 [arXiv:2008.07543] [INSPIRE].

[59] G.J. Turiaci and A. Zhiboedov, Veneziano amplitude of Vasiliev theory, JHEP 10 (2018) 034 [arXiv: 1802.04390] [INSPIRE].

[60] R. Yacoby, Scalar correlators in bosonic Chern-Simons vector models, arXiv:1805.11627 [INSPIRE].

[61] V.K. Dobrev, G. Mack, V.B. Petkova, S.G. Petrova and I.T. Todorov, Harmonic analysis on the $n$-dimensional Lorentz group and its application to conformal quantum field theory, Lect. Notes Phys. 63 (1977) 1 [inSPIRE].

[62] S. Jain, R.R. John and V. Malvimat, Constraining momentum space correlators using slightly broken higher spin symmetry, JHEP 04 (2021) 231 [arXiv: 2008.08610] [INSPIRE].

[63] S.D. Chowdhury, A. Gadde, T. Gopalka, I. Halder, L. Janagal and S. Minwalla, Classifying and constraining local four photon and four graviton S-matrices, JHEP 02 (2020) 114 [arXiv: 1910.14392] [INSPIRE].

[64] K. Inbasekar, S. Jain, V. Malvimat, A. Mehta, P. Nayak and T. Sharma, Correlation functions in $N=2$ supersymmetric vector matter Chern-Simons theory, JHEP 04 (2020) 207 [arXiv: 1907.11722] [INSPIRE].

[65] S. Jain, M. Mandlik, S. Minwalla, T. Takimi, S.R. Wadia and S. Yokoyama, Unitarity, crossing symmetry and duality of the S-matrix in large $N$ Chern-Simons theories with fundamental matter, JHEP 04 (2015) 129 [arXiv: 1404.6373] [INSPIRE].

[66] K. Inbasekar, S. Jain, S. Mazumdar, S. Minwalla, V. Umesh and S. Yokoyama, Unitarity, crossing symmetry and duality in the scattering of $N=1$ SUSY matter Chern-Simons theories, JHEP 10 (2015) 176 [arXiv:1505.06571] [INSPIRE].

[67] G. Gur-Ari, S.A. Hartnoll and R. Mahajan, Transport in Chern-Simons-matter theories, JHEP 07 (2016) 090 [arXiv:1605.01122] [INSPIRE]. 